\title{
Distribución espacial y temporal de la salinidad en la columna de agua del sector estuarino del Humedal Nacional Térraba Sierpe, Costa Rica
}

\author{
Jorge Picado Barboza \\ Proyecto Hidroeléctrico El Diquís, Instituto Costarricense de Electricidad, Costa Rica; jpicado@ice.go.cr \\ Recibido 11-VIII-2014. Corregido 20-XI-2014. Aceptado 22-XII-2014.
}

\begin{abstract}
Spatial and temporal salinity distribution in the water column of the estuarine sector of the Térraba Sierpe National Wetland, Costa Rica. The analysis of salinity data collected during three campaigns of monitoring (2002-2004, 2005-2008 and 2011-2012) in the estuarine sector of Térraba Sierpe National Wetlands (HNTS) was performed. The information collected allows to separate a northern zone with a strong influence of the Río Grande de Térraba, which during the period of low flow (December-April) shows characteristics of a partially mixed estuary, while it resembles a salt-wedge estuary during intermediate and high flows rates (May-November). A central area with greater marine influence features a vertically homogeneous or well mixed estuary, while the south of the estuary has a balanced (intermediate) condition, through the contributions of the Sierpe river and the tidal regime, which confers characteristics of a partially mixed estuary. The statistical analysis model confirms variations in the salinity values in relation to tides, time of year (season) and the distance along a longitudinal profile of $24 \mathrm{~km}$ away from the town of Ciudad Cortés to the sea opening at Coronado mouth $\left(\mathrm{F}_{1,47}=2.5, \mathrm{p}<0.001\right)$. The first $7 \mathrm{~km}$ downstream from Ciudad Cortes have a very well-defined fluvial condition with salinities below $1 \mathrm{psu}$, in the sector of Viuda island, located $8 \mathrm{~km}$ towards the sea, there is a range of variation in salinity values higher than $5 \mathrm{psu}$; a final section that starts $14 \mathrm{~km}$ towards the Coronado mouth shows a gradual increase in the salinity values up to 30 psu reflecting a greater influence of the tides. The eventual development of the El Diquis hydroelectric project could cause an increase in the contribution of freshwater between an $82-66 \%$ of the minimum and maximum average flow values over the northern part of the estuarine sector of the HNTS during the low flow periods, which would create similar conditions to those recorded during the intermediate flow rates. The provided analysis constitutes a first approach to the understanding of the estuarine dynamics of the HNTS. Analysis and monitoring of the relationships between the physical, chemical and biological aspects are essential for future discern between possible sources of changes in the estuarine sector of the HNTS and must be considered to be a firm commitment by the institutions involved in the management and conservation of this ecosystem. Rev. Biol. Trop. 63 (Suppl. 1): 75-96. Epub 2015 April 01.
\end{abstract}

Key words: Salinity, estuary, wetland, Térraba, Sierpe, Diquís.

El gradiente de salinidad es una de las principales características de un ecosistema estuarino, y su distribución es el resultado de la interacción entre aguas dulces y saladas a través de diferentes procesos dinámicos asociados principalmente a los cambios en las mareas (amplitud mareal) y la cantidad de agua dulce aportada por los ríos (caudal) (Mann \& Lazier, 1996; Telesh \& Khlebovich, 2010). Ambos factores varían a diferentes escalas temporales; las mareas, por ejemplo, responden a fenómenos astronómicos producidos por la fuerza de atracción gravitacional de la luna y el sol, cuya manifestación local está relacionada con aspectos océano-meteorológicos como la extensión y profundidad de la plataforma continental, presiones atmosféricas y corrientes marinas, entre otros (Svendsen, 1997; Talley, 2002; Telesh \& Khlebovich, 2010). Por su parte, los caudales se asocian principalmente con variaciones estacionales en la precipitación, como por ejemplo las épocas seca y lluviosa de la costa del Pacífico Sur de Costa Rica (Lizano, 2006). De esta manera en los estuarios se observan variaciones 
diarias en la salinidad con el cambio entre la marea alta y baja, magnificaciones mensuales con las mareas vivas y extraordinarias, así como aquéllas producidas por fenómenos climáticos que ocurren en periodos o ciclos más amplios, como por ejemplo los fenómenos de El Niño y La Niña, huracanes y tormentas tropicales (Lizano, 1997; Talley, 2002; Stott et al., 2004; Lizano, 2006).

El agua de origen marino posee una mayor densidad que el agua dulce, esto como producto de las sales disueltas en ella, lo cual produce que el agua salada se hunda debajo de la dulce y adquiera la forma de una "cuña" que incursiona y se retira del estuario al ritmo de las mareas y que puede internarse río arriba, en algunos casos hasta varias decenas de kilómetros (Mann \& Lazier, 1996; Talley, 2002; Gómez, 2006). Estos procesos dinámicos modifican las características físicas y químicas del agua y el valor de salinidad de un espacio en un momento específico del estuario, este ámbito de variaciones en la salinidad es uno de los factores determinantes sobre la distribución y abundancia de la flora y fauna en los ecosistemas estuarinos; relación que ha sido ampliamente documentada (Robertson \& Alongi, 1992; Jiménez, 1994; Lalli \& Parsons, 2000).

Los estudios de las características físicas y químicas de los estuarios de la costa Pacífica de Costa Rica se han concentrado principalmente en los Golfos de Nicoya, Papagayo y Dulce, así como algunos esteros como Puntarenas y Golfito (Voorhis, Epifanio, Maurer, Dittel \& Vargas, 1983; Lizano \& Vargas, 1993; Brenes, León \& Chaves, 2001; Acuña, Vargas \& Córdoba, 2006; Silva \& Acuña, 2006; Svendsen et al., 2006), pero muy pocos se han desarrollado en la Bahía de Coronado y en especial en el sector estuarino del delta del Río Grande de Térraba y Río Sierpe. Con el objetivo de describir la variación espacial y temporal de la salinidad en la columna de agua de los principales esteros del sector estuarino del Humedal Nacional Térraba Sierpe (HNTS), el Instituto Costarricense de Electricidad (ICE), a través del Estudio de Impacto Ambiental (EsIA) del Proyecto Hidroeléctrico El Diquís
(PHED), ha desarrollado un programa de monitoreo de las características físicas y químicas del agua en este ecosistema. Este monitoreo pretende proporcionar información base para la evaluación de los cambios potenciales derivados de la modificación del régimen de caudales que ingresan al HNTS mediante el Río Grande de Térraba.

\section{MATERIALES Y MÉTODOS}

Área de Estudio: La cuenca del Río Grande de Térraba es una de las más grandes del país, representando cerca del $10 \%$ de la superficie continental, la precipitación media anual va desde los 1500 a los $6000 \mathrm{~mm}$, en las partes altas se registra entre los 1500 y los $3000 \mathrm{~mm}$, en la parte media se registran lluvias de 3000 a $4000 \mathrm{~mm}$ y en la parte baja de la cuenca de $4000 \mathrm{~mm}$, los meses de setiembre y octubre suelen ser los más lluviosos, aportando aproximadamente un $13 \%$ y $19 \%$, respectivamente, de la precipitación promedio anual (Rojas, 2011).

Los humedales de la cuenca baja de los ríos Térraba y Sierpe se ubican en una sección continental y costera del Pacífico Sur de Costa Rica correspondiente al Valle del Diquís, el sistema está delimitado por el océano Pacífico, la fila Brunqueña y la serranía de la península de Osa (Bravo \& Ocampo, 1993; Fonseca, Alvarado, Cortés \& Herrera, 2009). La mayor parte de estos humedales constituyen el Humedal Nacional Térraba Sierpe (HNTS), área protegida por el sistema de áreas de Conservación de Costa Rica y que comprende una superficie aproximada de $33031.8 \mathrm{ha}$, de las cuales un $56 \%$ corresponden a ecosistemas estuarinos (Fig. 1). El HNTS por sus características ecológicas, biológicas y los aspectos socio-económicos ligados a sus manglares, ha sido reconocido dentro del grupo de humedales más importantes del país y a nivel internacional como sitio Ramsar desde 1995 (Bravo \& Ocampo, 1993; Jiménez, 1994; Cordero, 2000; Umaña, Bolaños, Mora, Pereira \& Gómez, 2000; Kapelle, Acevedo, González \& Monge, 2002; Sierra Vartanián \& Polimeni, 2006). 

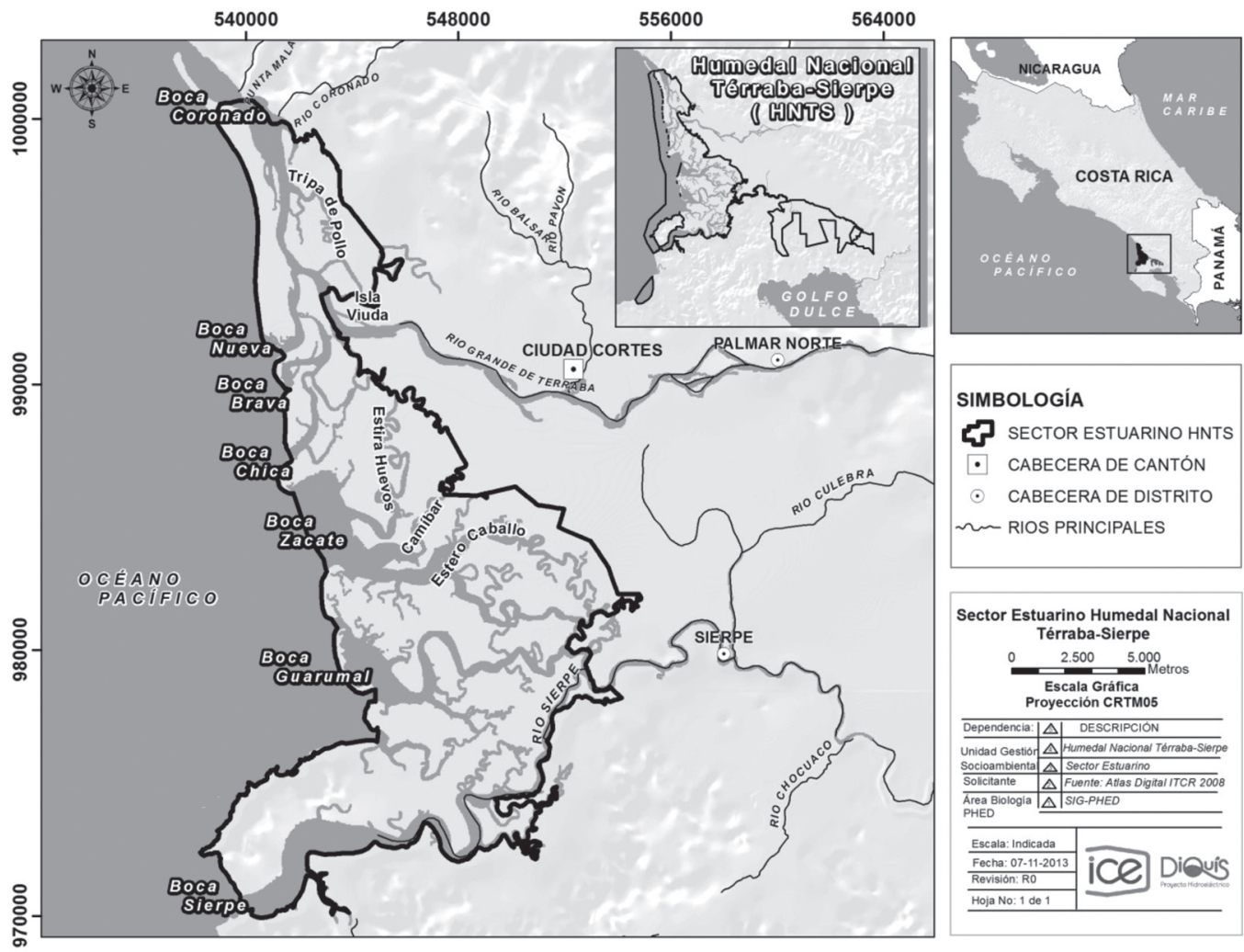

Fig. 1. Ubicación del sector estuarino del Humedal Nacional Térraba Sierpe. Área de Conservación Osa. Costa Rica. Fuente: Área de Biología, ICE-PHED, 2013.

Fig. 1. Térraba Sierpe National Wetland estuarine sector. Área de Conservación Osa. Costa Rica. Source: Área de Biología, ICE-PHED, 2013.

Medición de salinidad: Los datos analizados corresponden a las mediciones de salinidad realizadas por el ICE en el sector estuarino del HNTS entre el periodo 2006-2012. El trabajo se realizó en 15 estaciones de muestreo fijas y 90 estaciones alternas con una separación de $500 \mathrm{~m}$ sobre los canales y esteros principales del HNTS, lo anterior con el objetivo de cubrir sectores representativos del área de estudio durante los principales periodos de precipitación a lo largo del año: estación seca (diciembre-abril), transición (mayo-agosto) y lluviosa (setiembrenoviembre). En total se realizaron 6998 mediciones de salinidad, 2842 correspondientes a la marea alta y 4156 mediciones durante la marea baja, en el Cuadro 1 se muestra el número de mediciones realizadas en cada periodo y zona del estuario (interno, medio, externo). En cada sitio de medición se estableció una sección transversal entre los márgenes del cauce del río, estero o canal del estuario, en esta sección se realizaron mediciones en tres puntos, uno en la parte central del cauce, otro en la margen derecha y otro en la margen izquierda, en cada uno se midió el valor de salinidad en la superficie $(<50 \mathrm{~cm}$ de profundidad) y en el fondo el cual dependía de la profundidad máxima del sitio. Las mediciones corresponden a unidades prácticas de salinidad (ups) mediante una sonda multiparámetros marca YSI modelo 556 MPS. Cuando se encontró una diferencia marcada entre la salinidad del fondo y la superficial, mayor a 10 ups, se realizó un perfil vertical con mediciones cada $50 \mathrm{~cm}$ de profundidad, 
CUADRO 1

Número de mediciones de la salinidad en el sector estuarino del Humedal Nacional Térraba Sierpe según año, periodo de precipitación y zona del estuario. ICE-PHED, periodo 2006-2012

TABLE 1

Number of salinity measurements in the estuarine sector of the Térraba Sierpe National Wetland according to year, precipitation period and estuarine zonation. ICE-PHED, period 2006-2012

\begin{tabular}{|c|c|c|c|c|c|c|c|c|c|c|c|c|c|c|}
\hline \multirow{2}{*}{ Año } & \multirow{2}{*}{ Estación } & \multicolumn{4}{|c|}{ Zona Norte } & \multicolumn{4}{|c|}{ Zona Central } & \multicolumn{4}{|c|}{ Zona Sur } & \multirow{2}{*}{ Total } \\
\hline & & $\mathrm{E}^{1}$ & $\mathrm{M}^{2}$ & $\mathrm{I}^{3}$ & Total & $E^{1}$ & $\mathrm{M}^{2}$ & $\mathrm{I}^{3}$ & Total & $\mathrm{E}^{1}$ & $\mathrm{M}^{2}$ & $\mathrm{I}^{3}$ & Total & \\
\hline 2006 & Seca & 65 & 75 & 1 & 141 & - & - & - & - & - & - & - & - & 141 \\
\hline \multirow[t]{3}{*}{2007} & Seca & 106 & 79 & 6 & 191 & 10 & 14 & 22 & 46 & - & - & 28 & 28 & 265 \\
\hline & Lluviosa & 150 & 225 & 13 & 388 & 85 & 169 & 137 & 391 & 12 & 46 & 80 & 138 & 917 \\
\hline & Transición & 168 & 462 & 9 & 639 & 82 & 301 & 245 & 628 & - & 8 & 87 & 95 & 1362 \\
\hline \multirow[t]{3}{*}{2008} & Seca & 117 & 157 & 2 & 276 & 4 & 34 & - & 38 & 30 & 60 & 76 & 166 & 480 \\
\hline & Lluviosa & 64 & 123 & 2 & 189 & 22 & 70 & 64 & 156 & 18 & 50 & 18 & 86 & 431 \\
\hline & Transición & 108 & 104 & 25 & 237 & 87 & 121 & 47 & 255 & 58 & 97 & 101 & 256 & 748 \\
\hline 2009 & Seca & 80 & 96 & 2 & 178 & 30 & 86 & 64 & 180 & - & - & 86 & 86 & 444 \\
\hline \multirow[t]{3}{*}{2012} & Seca & 291 & 885 & 166 & 1342 & 64 & 40 & - & 104 & - & - & - & - & 1446 \\
\hline & Transición & 201 & 473 & 87 & 761 & 3 & - & - & 3 & - & - & - & - & 764 \\
\hline & Total & 1350 & 2679 & 313 & 4342 & 387 & 835 & 579 & 1801 & 118 & 261 & 476 & 855 & 6998 \\
\hline
\end{tabular}

$\mathrm{E}^{1}=$ Estuario externo, $\mathrm{M}^{2}=$ Estuario medio, $\mathrm{I}^{3}=$ Estuario interno.

esto fue realizado en los sitios donde hay un encuentro de las masas de agua dulce y salada, conocidos popularmente por los pescadores como golpes o topes de agua marina y dulce. Los datos fueron analizados utilizando herramientas exploratorias multivariables como el análisis de conglomerados y escalamiento multidimensional, además se realizaron análisis de varianzas para comprobar diferencias en la salinidad entre épocas, sitios y mareas; para realizar estos análisis se utilizaron los paquetes estadísticos Systat ${ }^{\circledR} 10$, Statistica ${ }^{\circledR} 6$ y PAST ${ }^{\circledR}$ (Clarke \& Warwick, 1994; Krebs, 1994; Zar, 1999; Hammer, Harper \& Ryan, 2001; Pla \& Matteucci, 2001).

Caudales: Utilizando la información del Área de Hidrología del ICE y tomando como referencia los registros de caudales en el Río Grande de Térraba en la estación hidrológica de Palmar Norte (estación 31-01) entre los años 1970 y 2010 , se establecieron tres periodos anuales de caudales: caudales mínimos o bajos, los cuales se registraron durante la época seca entre los meses de diciembre y abril, cuyo caudal promedio fue de $115 \mathrm{~m}^{3} / \mathrm{s}$; caudales intermedios, los cuales correspondieron al periodo de transición entre la estación seca y lluviosa entre los meses de mayo y agosto, cuyo valor promedio fue de $335 \mathrm{~m}^{3} / \mathrm{s}$; y finalmente caudales máximos o altos que correspondieron a los meses de mayor precipitación entre setiembre y noviembre, con un caudal promedio de $615 \mathrm{~m}^{3} / \mathrm{s}$ (Fig. 2).

\section{Elaboración de modelos de distribución} de la salinidad: Mediante la colaboración del departamento Sistemas de Información Geográfica del PHED, se procedió a realizar una interpolación digital de los datos de salinidad por medio de la herramienta Spatial Analyst Tools/Spline with Barriers. El "Spline with Barriers" es un método de interpolación de uso genérico que ajusta una superficie de curvatura mínima a través de los puntos ingresados, es adecuado para mostrar variaciones graduales de superficies como por ejemplo elevaciones o concentraciones de contaminación. Para el análisis de la salinidad se utilizó la incorporación de barreras (por ejemplo riscos, cavidades, $\mathrm{u}$ otros objetos que interrumpen la continuidad de los valores analizados) las cuales pueden ser 


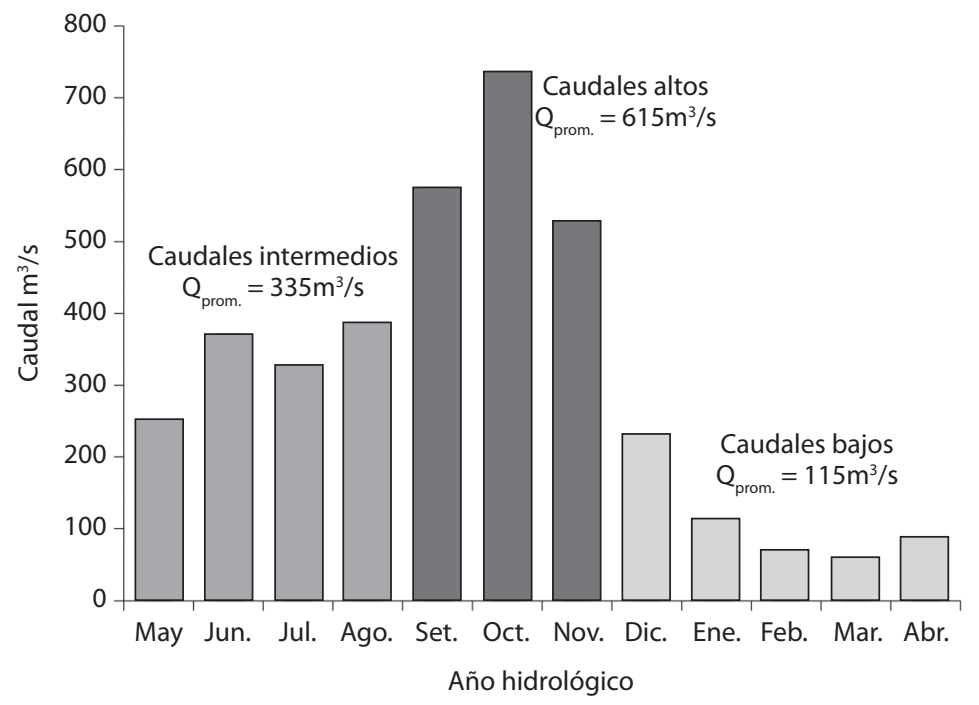

Fig. 2. Hidrograma del Río Grande de Térraba en la estación hidrológica 32-01 ubicada en Palmar Norte, Puntarenas, Costa Rica. Clasificación basada en caudales promedio mensuales, periodo de registro 1970-2010. Área de Hidrología del ICE, 2013.

Fig. 2. Grande de Térraba river hydrogram at gauging station 32-01 located at Palmar Norte, Puntarenas, Costa Rica. Classification base on monthly average flow values, registered period 1970-2010. Hydrology Department of ICE, 2013.

líneas o polígonos, es decir, se limitó la interpolación de los datos a una cobertura de esteros y canales en el humedal.

Con el objetivo de analizar los procesos de intrusión salina sobre el sector estuarino bajo la influencia directa del Río Grande de Térraba, se procedió a construir un perfil de profundidad, salinidad y distancia desde Ciudad Cortés, el cual es el punto de referencia continental aguas arriba hasta donde se han observado cambios relevantes en los niveles del Río Grande de Térraba con las mareas (Picado, Lara, Castro \& Barrantes, 2008), y 24km lineales sobre el cauce del río Grande de Térraba y canal principal del estuario hasta la desembocadura al mar en Boca Coronado. Estos perfiles fueron creados utilizando la herramienta "3D Contour Plot-Spline Proyection" del software Statistica versión $7 \AA$.

\section{RESULTADOS}

Los datos recopilados muestran diferencias en la distribución de la salinidad como resultado de la interacción entre las mareas, la época del año y la distancia desde Ciudad Cortés hasta la desembocadura al mar $\left(\mathrm{F}_{1,47}=2.5\right.$, $\mathrm{p}<0.001)$. A continuación se describe la distribución de la salinidad en cada uno de los tres periodos del año hidrológico propuestos para la cuenca del Río Grande de Térraba en el presente estudio.

Caudales bajos: En este periodo los caudales promedio mensuales registrados en el Río Grande de Térraba variaron entre 60 y $230 \mathrm{~m}^{3} / \mathrm{s}$, el caudal promedio de este periodo fue cercano a los $115 \mathrm{~m}^{3} / \mathrm{s}$ (Fig. 2). Durante las mareas altas en la zona norte, la cual se encuentra bajo la influencia directa del Río Grande de Térraba, el valor promedio de salinidad en el fondo y superficie fue de 14 ups \pm 11 ups, lo cual reflejó una alta variabilidad de valores de salinidad en este zona, mientras que la zona central, que comprende los esteros y desembocaduras de Zacate y Guarumal, presentó valores promedio de 25ups $\pm 3-5$ ups, indicando una mayor estabilidad e influencia marina en los valores 
de salinidad. Por su parte en el extremo sur, que corresponde al estero Sierpe, los valores promedio correspondieron a 17 ups \pm 10 ups en superficie y fondo, mostrando un leve descenso con respecto a la zona central y el cual se puede atribuir a los aportes de agua dulce del Río Sierpe (Fig. 3A, B, Cuadro 2).
Con las mareas bajas en la zona norte se observaron nuevamente valores muy variables que comprenden entre 13ups \pm 11 ups en superficie y 15 ups \pm 12 ups en el fondo. La zona central mantuvo valores superiores a 20 ups \pm 5 ups tanto en fondo como en superficie, mientras que en el extremo sur los valores promedio fueron de

\section{CUADRO 2}

Valores mínimos, máximos, promedio y desviación estándar de la salinidad registrada en tres zonas el sector estuarino del Humedal Nacional Térraba Sierpe durante tres periodos del año y ciclo de marea. ICE-PHED, periodo 2006-2012

\section{TABLE 2}

Mimum, maximum, average and standard deviation of salinity values in three zones of the estuarine sector of the TérrabaSierpe National Wetland during three periods of the year and tidal cycle. ICE-PHED, period 2006-2012

\begin{tabular}{|c|c|c|c|c|c|c|c|c|}
\hline \multirow{3}{*}{ Sector * } & \multirow{3}{*}{ Zona } & & \multicolumn{6}{|c|}{ Periodo/ciclo de marea } \\
\hline & & & \multicolumn{2}{|c|}{ Diciembre-abril } & \multicolumn{2}{|c|}{ Mayo-agosto } & \multicolumn{2}{|c|}{ Setiembre-noviembre } \\
\hline & & & Alta & Baja & Alta & Baja & Alta & Baja \\
\hline \multirow[t]{12}{*}{ Norte } & \multirow[t]{4}{*}{ Externa } & Máx. & 32.0 & 32.1 & 30.6 & 33.2 & 26.7 & 9.0 \\
\hline & & Mín. & 0.2 & 0.0 & 0.0 & 0.0 & 0.0 & 0.0 \\
\hline & & Prom. & 21.2 & 23.1 & 18.8 & 18.3 & 2.2 & 0.2 \\
\hline & & Desv. & 10.2 & 8.6 & 11.9 & 12.9 & 5.4 & 0.9 \\
\hline & \multirow[t]{4}{*}{ Media } & Máx. & 32.4 & 30.9 & 29.8 & 31.8 & 7.0 & 1.3 \\
\hline & & Mín. & 0.0 & 0.0 & 0.0 & 0.0 & 0.0 & 0.0 \\
\hline & & Prom. & 12.1 & 13.1 & 4.1 & 5.3 & 0.2 & 0.0 \\
\hline & & Desv. & 10.1 & 10.9 & 6.7 & 7.8 & 0.7 & 0.2 \\
\hline & \multirow[t]{4}{*}{ Interna } & Máx. & 0.7 & 0.6 & 0.1 & 0.3 & 0.0 & 0.0 \\
\hline & & Mín. & 0.0 & 0.0 & 0.0 & 0.0 & 0.0 & 0.0 \\
\hline & & Prom. & 0.2 & 0.2 & 0.0 & 0.1 & 0.0 & 0.0 \\
\hline & & Desv. & 0.3 & 0.2 & 0.0 & 0.1 & 0.0 & 0.0 \\
\hline \multirow[t]{12}{*}{ Central } & \multirow[t]{4}{*}{ Externa } & Máx. & 30.4 & 32.1 & 30.0 & 30.3 & 30.6 & 21.0 \\
\hline & & Mín. & 10.0 & 23.4 & 14.8 & 0.0 & 7.0 & 0.0 \\
\hline & & Prom. & 26.0 & 30.1 & 26.5 & 15.7 & 22.3 & 7.8 \\
\hline & & Desv. & 6.3 & 2.6 & 3.5 & 7.7 & 7.7 & 6.8 \\
\hline & \multirow[t]{4}{*}{ Media } & Máx. & 30.0 & 31.6 & 29.1 & 25.5 & 30.6 & 15.0 \\
\hline & & Mín. & 16.0 & 12.1 & 0.4 & 0.0 & 1.0 & 0.0 \\
\hline & & Prom. & 27.2 & 26.1 & 20.6 & 12.1 & 15.4 & 4.5 \\
\hline & & Desv. & 2.3 & 4.3 & 6.5 & 7.4 & 9.9 & 4.0 \\
\hline & \multirow[t]{4}{*}{ Interna } & Máx. & 23.0 & 27.0 & 24.0 & 30.0 & 16.0 & 6.6 \\
\hline & & Mín. & 13.0 & 5.0 & 2.7 & 0.0 & 1.0 & 0.0 \\
\hline & & Prom. & 20.4 & 19.6 & 14.2 & 4.6 & 7.5 & 0.8 \\
\hline & & Desv. & 3.1 & 4.4 & 5.1 & 3.9 & 4.2 & 1.3 \\
\hline \multirow[t]{12}{*}{ Sur } & \multirow[t]{4}{*}{ Externa } & Máx. & 31.4 & 30.9 & 30.1 & 29.7 & 29.1 & 12.0 \\
\hline & & Mín. & 30.4 & 26.6 & 26.7 & 20.4 & 20.8 & 7.0 \\
\hline & & Prom. & 30.8 & 28.9 & 29.2 & 25.8 & 25.4 & 9.5 \\
\hline & & Desv. & 0.2 & 1.4 & 1.0 & 2.3 & 3.0 & 2.0 \\
\hline & \multirow[t]{4}{*}{ Media } & Máx. & 29.2 & 25.9 & 28.4 & 25.4 & 28.8 & 15.0 \\
\hline & & Mín. & 20.9 & 13.1 & 10.9 & 3.9 & 4.9 & 1.0 \\
\hline & & Prom. & 25.9 & 20.1 & 20.0 & 16.4 & 18.8 & 3.4 \\
\hline & & Desv. & 2.6 & 4.2 & 4.7 & 6.7 & 5.1 & 2.9 \\
\hline & \multirow[t]{4}{*}{ Interna } & Máx. & 20.0 & 20.1 & 14.0 & 3.1 & 14.8 & 1.0 \\
\hline & & Mín. & 1.0 & 0.0 & 0.0 & 0.0 & 0.7 & 0.0 \\
\hline & & Prom. & 9.6 & 4.1 & 1.7 & 0.3 & 4.8 & 0.1 \\
\hline & & Desv. & 6.1 & 4.7 & 3.0 & 0.8 & 3.2 & 0.3 \\
\hline
\end{tabular}

*Norte: influencia directa del Río Grande de Térraba; Central: esteros y desembocadura de boca Zacate; Sur: influencia directa del Río Sierpe. 

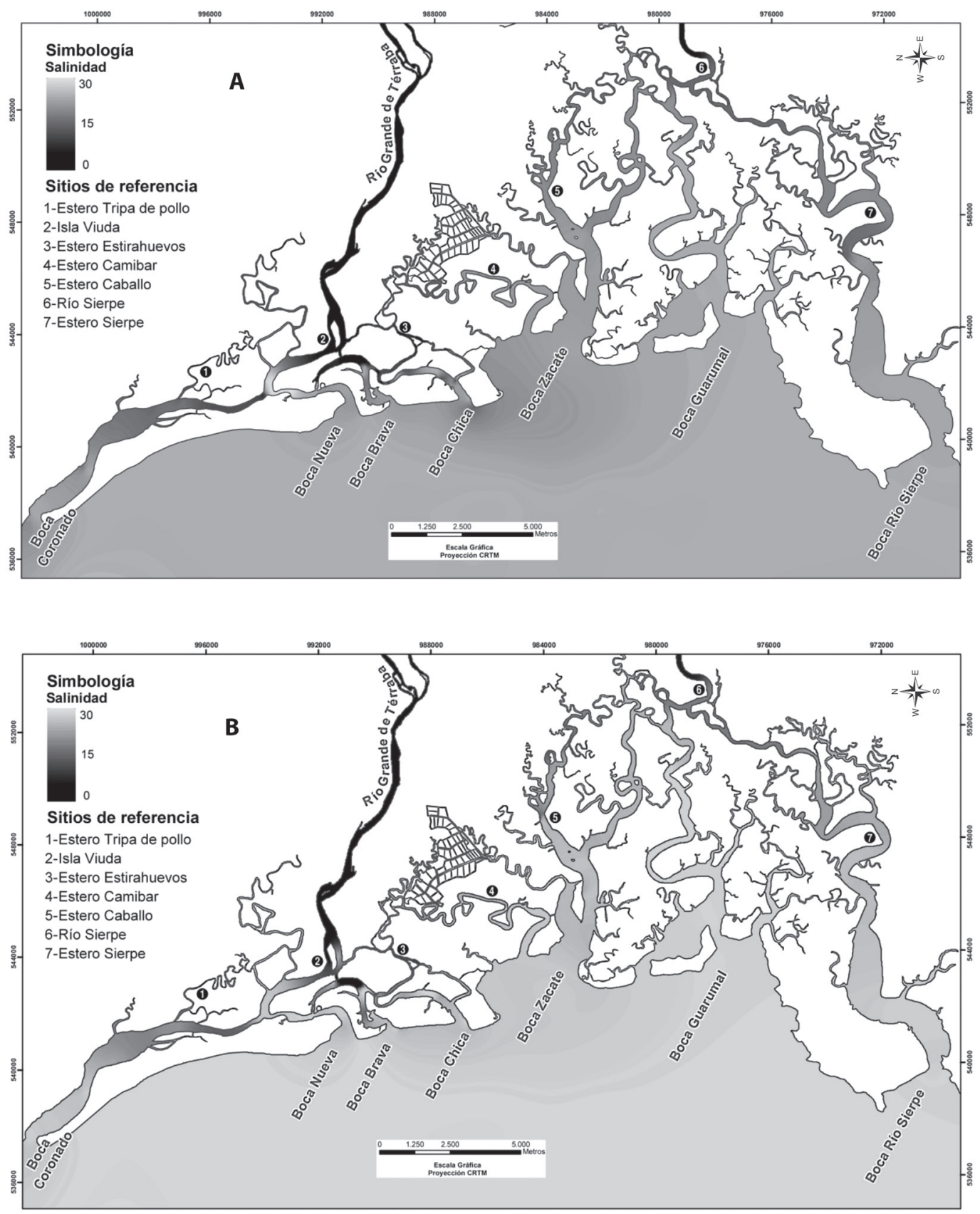

Fig. 3. Modelo de distribución de la salinidad en: A) superficie $(<50 \mathrm{~cm})$, B) profundidad máxima de los esteros durante la marea alta en el periodo de caudales bajos (diciembre-abril). Sector estuarino Humedal Nacional Térraba-Sierpe, ICEPHED, periodo 2006-2012.

Fig. 3. Salinity distribution model: A) surface $(<50 \mathrm{~cm})$, B) maximum channel depth during high tide and low flows (December-April). Terraba Sierpe National Wetland Estuarine sector, ICE-PHED, period 2006-2012. 
10ups \pm 9 ups, reflejando una alta variabilidad en los valores de salinidad. Como es de esperar los valores bajos de salinidad durante la marea baja se asociaron a las partes internas de los esteros y se observó el incremento de los valores hacia las desembocaduras, especialmente en las zonas norte y sur del sector estuarino del HNTS (Fig. 4A, B, Cuadro 2).

Caudales intermedios: Durante este periodo los caudales promedio mensuales registrados en el Río Grande de Térraba varían entre 250 y $390 \mathrm{~m}^{3} / \mathrm{s}$, el valor promedio de este periodo es cercano a los $335 \mathrm{~m}^{3} / \mathrm{s}$ (Fig. 2), correspondiendo a caudales similares a los que se esperarían con la variación en el régimen hidrológico producido por la operación del P.H. El Diquís durante los meses de verano. En términos generales durante este periodo se produce una mayor diferencia de salinidad entre las capas de agua del fondo y superficie como resultado del aumento en los aportes del agua dulce. En el extremo norte durante la marea alta la salinidad promedio en el fondo es de 10ups y en la superficie de 8ups, nuevamente con una alta variabilidad que abarca valores desde 0ups hasta 30ups, mientras que en la zona central los valores promedio en fondo y superficie comprenden 20ups y 18 ups \pm 7 ups. Por su parte en el extremo sur se registró un valor promedio de 10ups y 11ups para el fondo y superficie también con una alta variabilidad que contempla valores des 0ups hasta 30ups. Como es de esperar la salinidad en superficie disminuye con respecto al periodo anterior. A pesar de esto el efecto sobre el sector central no es tan fuerte y mantiene una mayor influencia marina en la mayoría de sus canales y esteros (Fig. 5A, B, Cuadro 2).

Durante los periodos de marea baja, se tornó evidente la influencia del Río Grande de Térraba en la zona norte del HNTS, esta disminución en la salinidad alcanza las bocas Brava, Nueva, Chica y una parte de Boca Zacate, los valores promedio en fondo y superficie alcanzan 10ups y 6ups y comprendiendo valores desde 0ups hasta 30ups. En la zona central el promedio se mantiene entre 10ups y 11ups en fondo y superficie pero mostrando una variabilidad en los valores de \pm 7 ups. Por su parte el extremo sur también presenta valores promedio de 10ups y 11ups pero con una variabilidad similar a la encontrada en el extremo norte. Durante este periodo se observa una disminución en la salinidad en la parte interna de los esteros Zacate, Caballo, Guarumal y Sierpe, en este caso por la influencia del Río Sierpe $\mathrm{y}$ algunos de los canales de riego del sector conocido como "las fincas" en el Valle del Diquís (Fig. 6A, B, Cuadro 2).

Caudales altos: Durante este periodo los caudales promedio mensuales registrados en el Río Grande de Térraba varían entre 530 y $740 \mathrm{~m}^{3} / \mathrm{s}$; el caudal promedio de este periodo es cercano a $\operatorname{los} 615 \mathrm{~m}^{3} / \mathrm{s}$ (Fig. 2). Con la marea alta en el extremo norte se registró tanto en el fondo como en la superficie un valor promedio de 1ups en el fondo y 0ups en superficie, con un valor máximo de 27 ups en el fondo y 10ups en superficie hacia la desembocadura en Coronado. En la zona central los valores promedio de salinidad comprenden 16ups y 12ups en el fondo y superficie con una desviación de \pm 10 ups y 9ups respectivamente. Por su parte el extremo sur presenta salinidades promedio entre 17ups y 12 ups en el fondo y superficie con una desviación de \pm 9 ups. Con el aumento en la precipitación y los altos volúmenes de agua que aportan los ríos, en especial el Río Grande de Térraba, se observa una fuerte influencia del agua dulce sobre la mayor parte del sector estuarino del HNTS, la disminución en la salinidad se presenta aún durante la marea alta en toda la columna de agua, en especial en los sectores bajo la influencia del Río Grande de Térraba (Fig. 7A, B, Cuadro 2).

Como es de esperar durante la marea baja se acrecienta aún más la situación descrita anteriormente, el extremo norte se registró tanto en el fondo como en la superficie un valor promedio de 0ups \pm 1 ups, con un valor máximo de 9ups en el fondo hacia la desembocadura en Coronado. En la zona central los valores promedio de salinidad comprenden 5ups y 2ups en el fondo y superficie con máximos de 

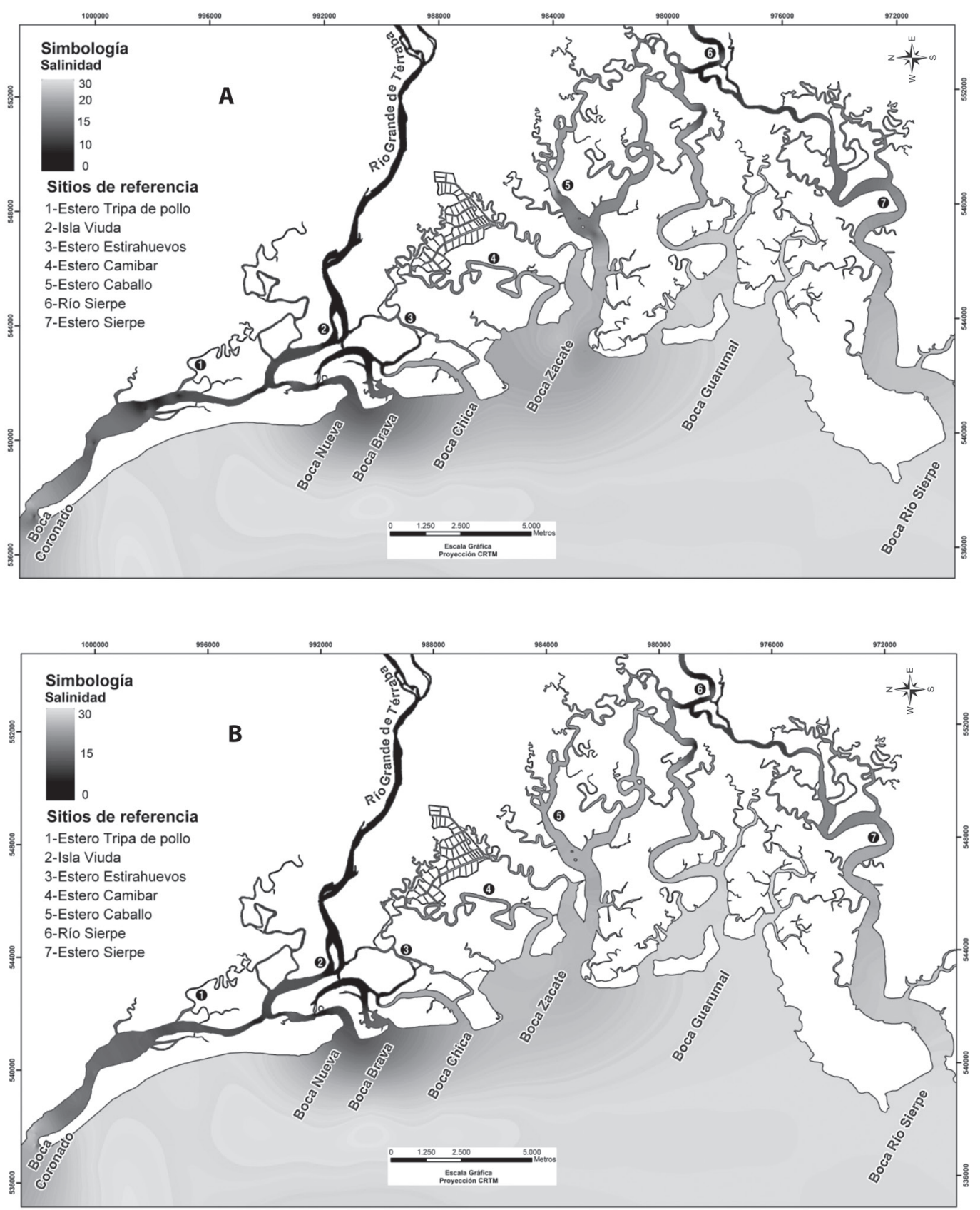

Fig. 4. Modelo de distribución de la salinidad en: A) superficie $(<50 \mathrm{~cm})$, B) profundidad máxima de los esteros durante la marea baja en el periodo de caudales bajos (diciembre-abril). Sector estuarino Humedal Nacional Térraba-Sierpe, ICEPHED, periodo 2006-2012.

Fig. 4. Salinity distribution model: A) surface $(<50 \mathrm{~cm})$, B) maximum channel depth during low tide and low flows (December-April). Terraba Sierpe National Wetland Estuarine sector, ICE-PHED, period 2006-2012. 

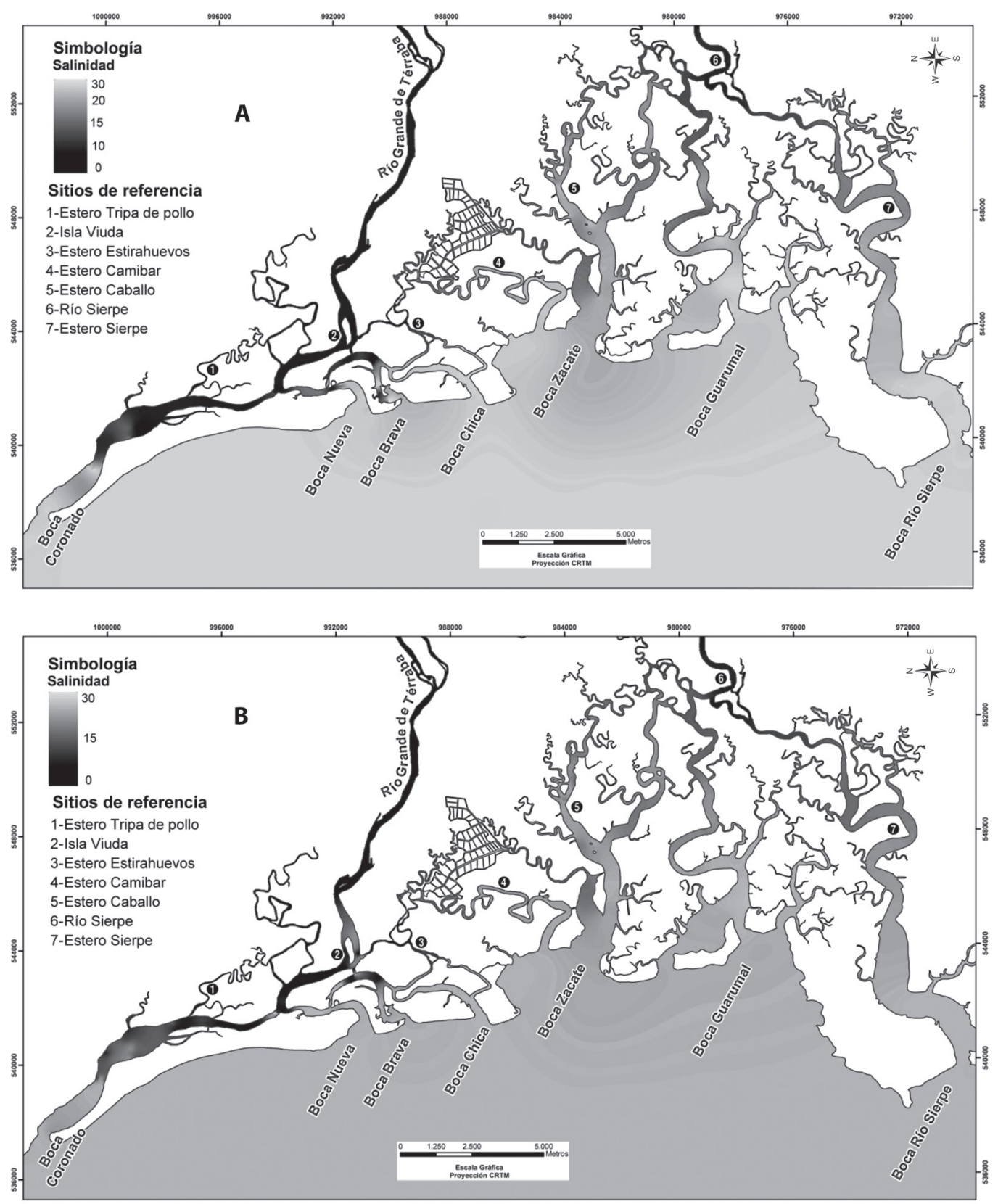

Fig. 5. Modelo de distribución de la salinidad en: A) superficie $(<50 \mathrm{~cm})$, B) profundidad máxima de los esteros durante la marea alta en el periodo de caudales intermedios (mayo-agosto). Sector estuarino Humedal Nacional Térraba-Sierpe, ICEPHED, periodo 2006-2012.

Fig. 5. Salinity distribution model: A) surface $(<50 \mathrm{~cm})$, B) maximum channel depth during high tide and transitional flows (may-august). Terraba Sierpe National Wetland Estuarine sector, ICE-PHED, period 2006-2012. 

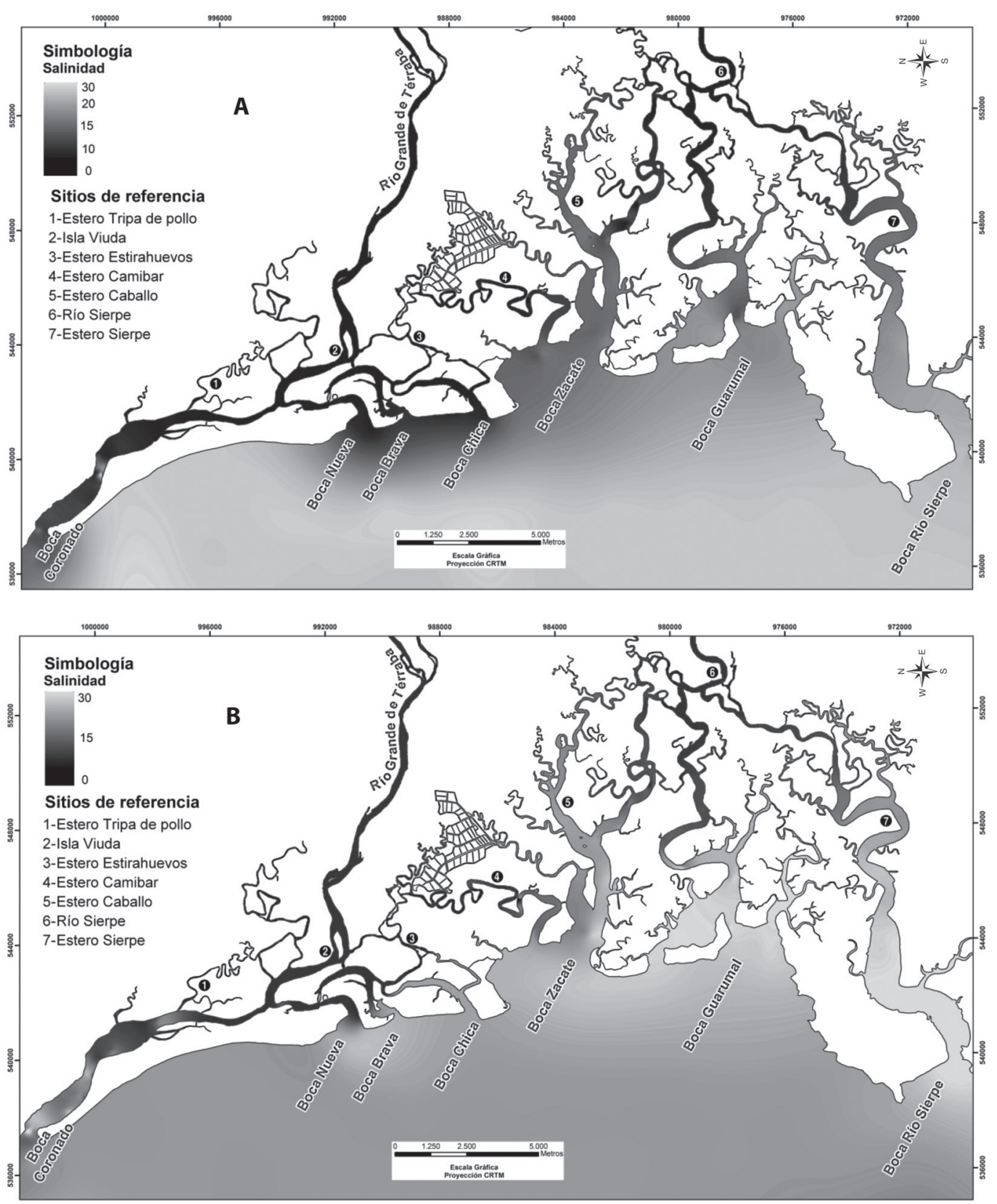

Fig. 6. Modelo de distribución de la salinidad en: A) superficie $(<50 \mathrm{~m})$, B) profundidad máxima de los esteros durante la marea baja en el periodo de caudales intermedios (mayo-agosto). Sector estuarino Humedal Nacional Térraba-Sierpe, ICEPHED, periodo 2006-2012.

Fig. 6. Salinity distribution model: A) surface $(<50 \mathrm{~cm})$, B) maximum channel depth during low tide and transitional flows (May-August). Terraba Sierpe National Wetland Estuarine sector, ICE-PHED, period 2006-2012. 

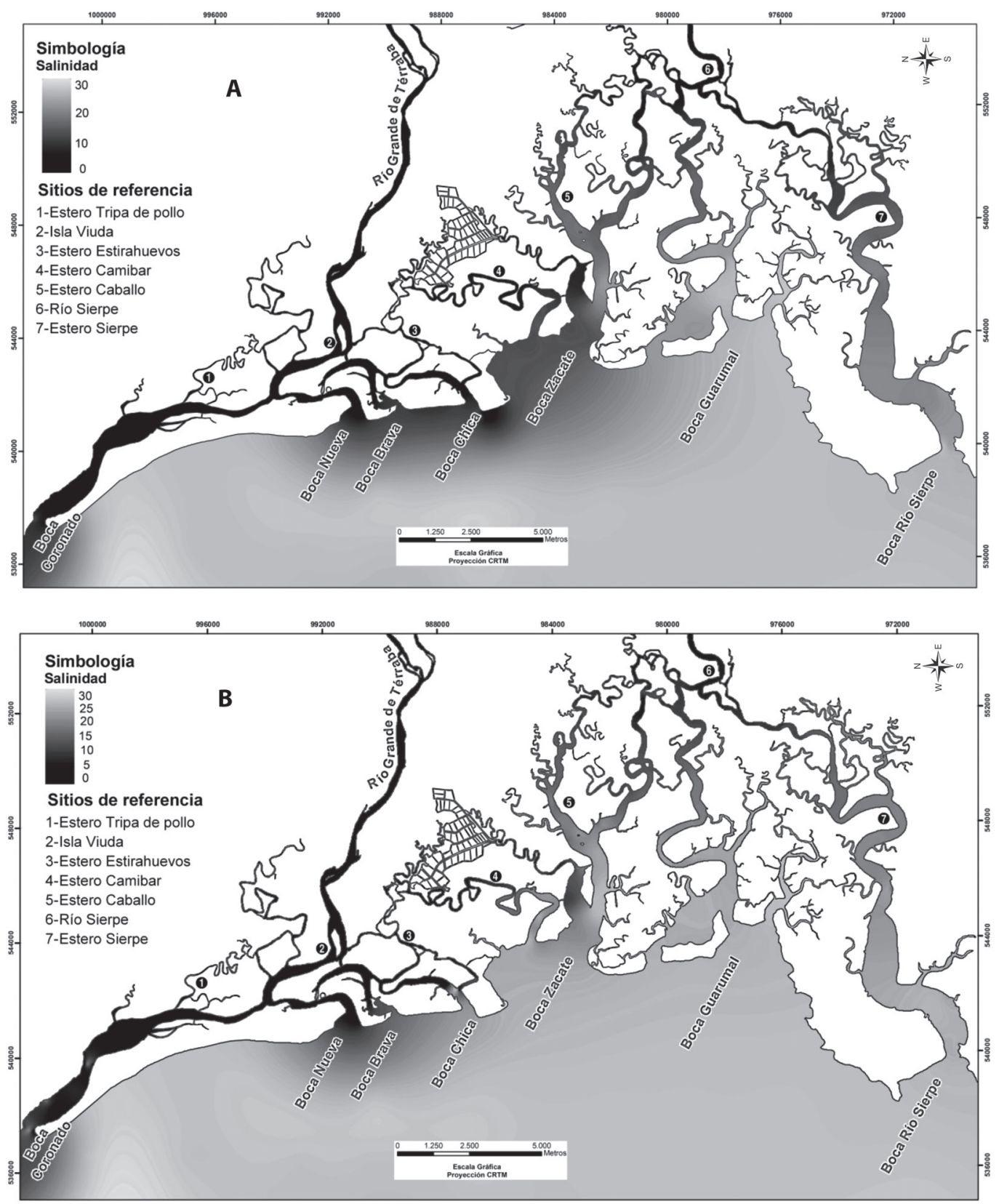

Fig. 7. Modelo de distribución de la salinidad en: A) superficie $(<50 \mathrm{~cm})$, B) profundidad máxima de los esteros durante la marea alta en el periodo de caudales altos (setiembre-noviembre). Sector estuarino Humedal Nacional Térraba-Sierpe, ICE-PHED, periodo 2006-2012.

Fig. 7. Salinity distribution model: A) surface $(<50 \mathrm{~cm})$, B) maximum channel depth during high tide and high flows (September-November). Terraba Sierpe National Wetland Estuarine sector, ICE-PHED, period 2006-2012. 
20ups hacia la desembocadura, mientras que el extremo sur presenta salinidades promedio entre 3ups y 2 ups con un máximo de 15 ups en el fondo y 8ups en superficie hacia la boca Sierpe. La influencia del agua dulce en la zona norte alcanza parte de Boca Zacate a través del canal Estirahuevos y los canales del sector conocido como la camaronera en Camíbar. Por su parte se observa que en las zonas central y sur estos cambios no son tan dramáticos, ya que conservan parte de la influencia marina encontrada en los otros periodos del año (Fig. 8A, B, Cuadro 2).

Perfil de salinidad: Los valores promedio, máximos y mínimos de la salinidad a lo largo del perfil longitudinal en el sector norte desde Ciudad Cortes hasta la desembocadura en boca Coronado, muestran que en términos generales los primeros $7 \mathrm{~km}$ aguas abajo de Ciudad Cortés mantienen una condición de baja salinidad durante todo el año; a partir del kilómetro ocho inicia la influencia de la salinidad con valores superiores a 5ups. En los kilómetros nueve y diez, que corresponde a los alrededores de la isla Viuda, se observa un incremento en los valores de salinidad, lo cual se atribuye a la entrada de marea por las bocas Nueva, Brava y Chica (Fig. 9). Por su parte el estero Tripa de Pollo contribuye con agua dulce y provoca un leve descenso en la salinidad en el kilómetros 11 y 12, mientras que la entrada de marea por la boca Nueva vuelve a incrementar los valores de salinidad en el kilómetro 13 (Fig. 9). A partir del kilómetro 14 se observa un gradiente de incremento de la salinidad hacia la Boca Coronado, el cual responde a una mayor influencia de las mareas y los procesos de mezcla con el agua dulce aportada por los pequeños ríos Coronado y Punta Mala. Resulta evidente además, la ampliación de la condición de agua dulce hasta prácticamente la salida al mar en el kilómetro 18 durante el periodo de grandes caudales del Río Grande de Térraba (Fig. 9).

Por su parte, el sector central mostró valores promedio más consistentes durante las diferentes épocas del año, a lo largo de los $14 \mathrm{~km}$ desde la parte interna del estero Caballo hasta la Boca Zacate, reflejando una mayor influencia marina y un régimen de distribución de la salinidad dominado por las mareas. En cuanto al sector sur se observó que los primeros seis kilómetros desde el poblado de Sierpe hacia el mar las salinidades son bajas y predomina un ambiente fluvial, a partir del kilómetro siete se evidencia un gradiente hacia una mayor salinidad en dirección a la Boca Sierpe y en los meses de mayor precipitación se refleja una disminución en los valores de salinidad, lo cual se enmarca en los cambios estacionales en el aporte de agua dulce por parte de la cuenca del Río Sierpe (Fig. 9).

La representación gráfica de la influencia de la salinidad en la columna del agua confirma que aproximadamente a partir de los $3 \mathrm{~km}$ aguas abajo de Ciudad Cortés inicia el registro de valores, aunque muy bajos, de salinidad que comprenden $<1$ ups registrados en el fondo del cauce (Fig. 10). Durante los meses de caudales bajos esta condición se mantiene hasta el kilómetro siete aguas abajo durante la marea alta, mientras que a partir del kilómetro nueve aguas abajo de Ciudad Cortés se presenta influencia de aguas marinas que producen valores de salinidad superiores a los 5ups en el fondo de los canales, desde este punto se observa un gradiente general de incremento en los valores de salinidad hacia la desembocadura, la distribución en la columna de agua refleja una buena mezcla entre las capas de agua dulce y salada (Fig. 10). Como es de esperar durante la marea baja prevalece la influencia del Río Grande de Térraba, provocando una disminución en los valores de salinidad especialmente en la superficie y que se prolonga hasta el kilómetro 17 aguas abajo por el canal principal hacia la boca Coronado (Fig. 10).

Durante el periodo de caudales intermedios y marea alta se evidencia la intrusión salina por el fondo de la columna del agua, los valores $>5$ ups se mantienen a partir de los $9 \mathrm{~km}$ aguas abajo de Ciudad Cortes, mientras que valores $>15$ ups predominan a partir de los $10 \mathrm{~km}$ aguas abajo (Fig. 10). Durante la marea baja se aprecia una fuerte influencia del agua dulce en la mayor parte del tramo evaluado, 

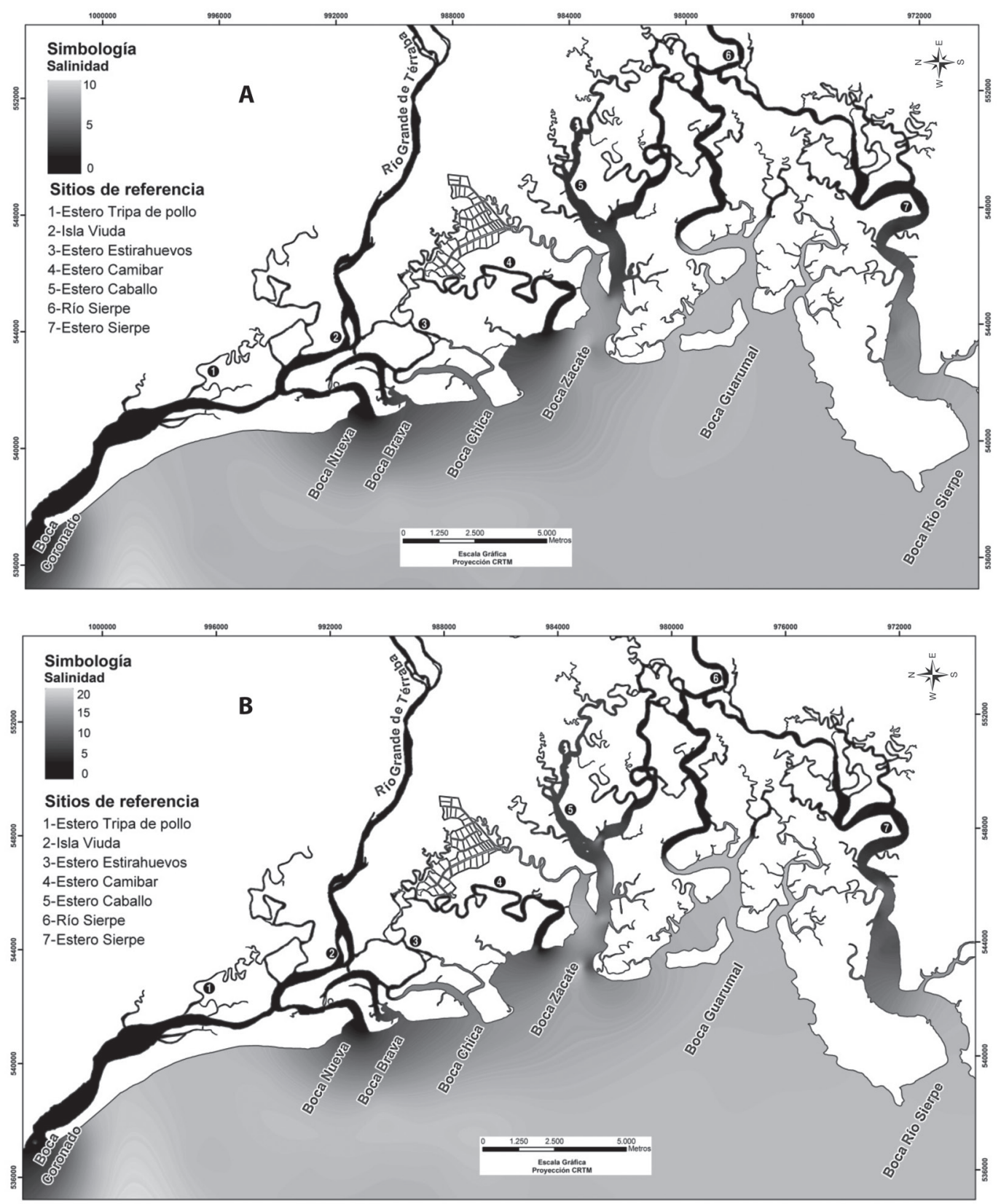

Fig. 8. Modelo de distribución de la salinidad en: A) superficie $(<50 \mathrm{~cm})$, B) profundidad máxima de los esteros durante la marea baja en el periodo de caudales altos (setiembre-noviembre). Sector estuarino Humedal Nacional Térraba-Sierpe, ICE-PHED, periodo 2006-2012.

Fig. 8. Salinity distribution model: A) surface $(<50 \mathrm{~cm})$, B) maximum channel depth during low tide and high flows (September-November). Terraba Sierpe National Wetland Estuarine sector, ICE-PHED, period 2006-2012. 


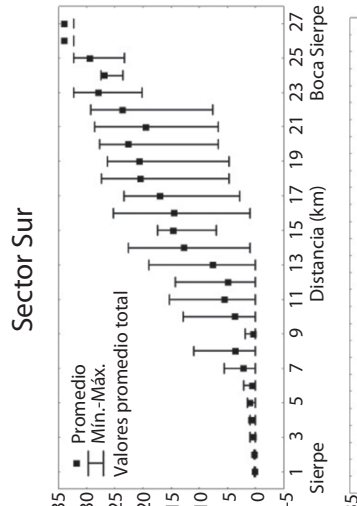

(sdn) pep!u!jes

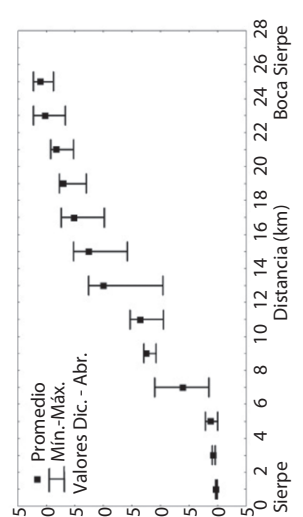

(sdn) pep!u!|es

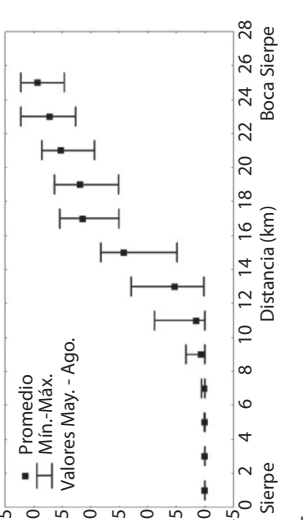

(sdn) pep!u!̣es

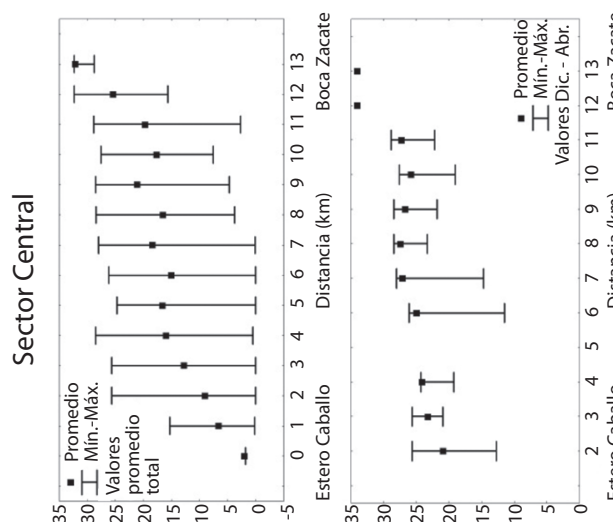

(sdn) pep!u!|es

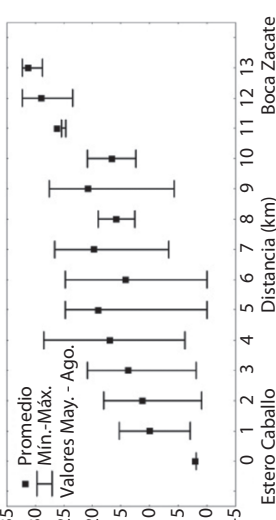

(sdn) pep!u!|es

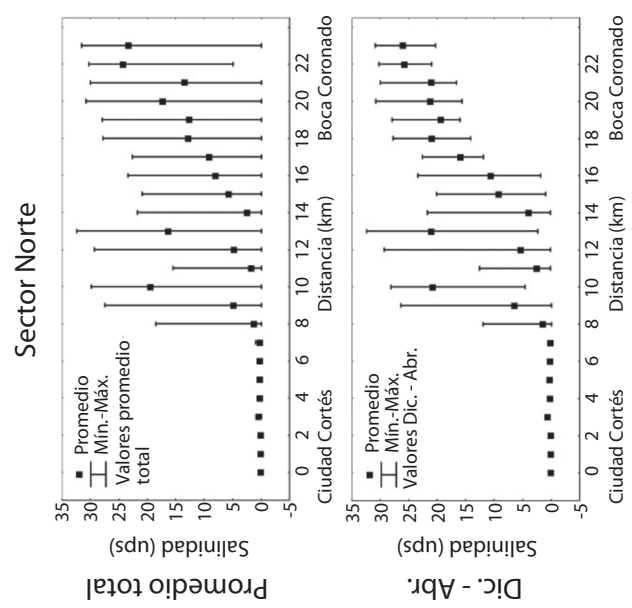

(sdn) pepiu!les

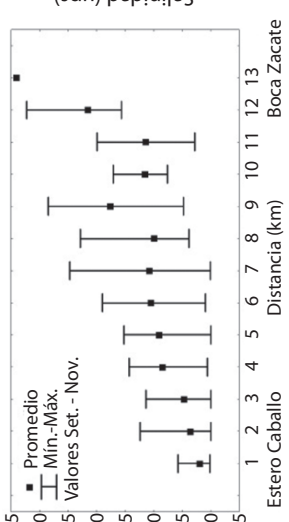

$m$ 을 으는 (sdn) pep!u!|es
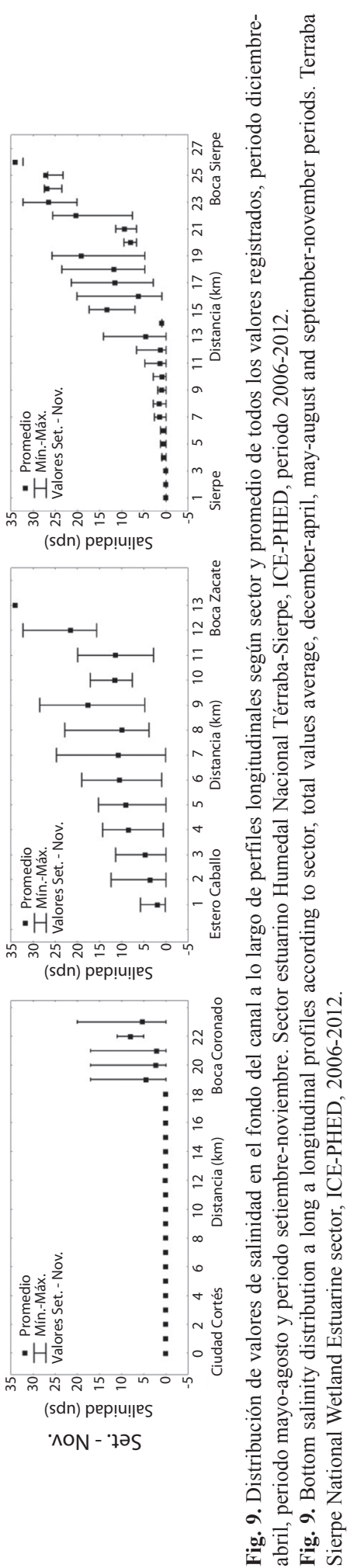

入

\&

.

每

व $\overline{0}$

贾

을

或苛

용

잉.

흐 후

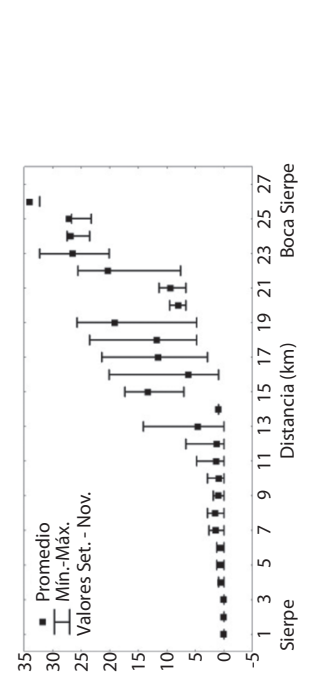

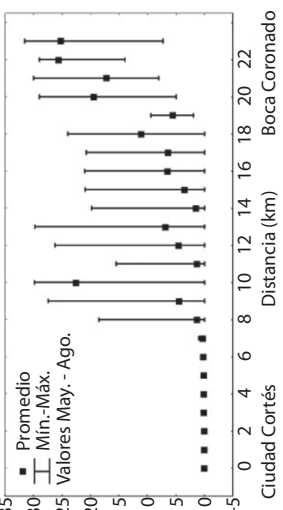

(sdn) pep!u!jes

oby - ·ew 

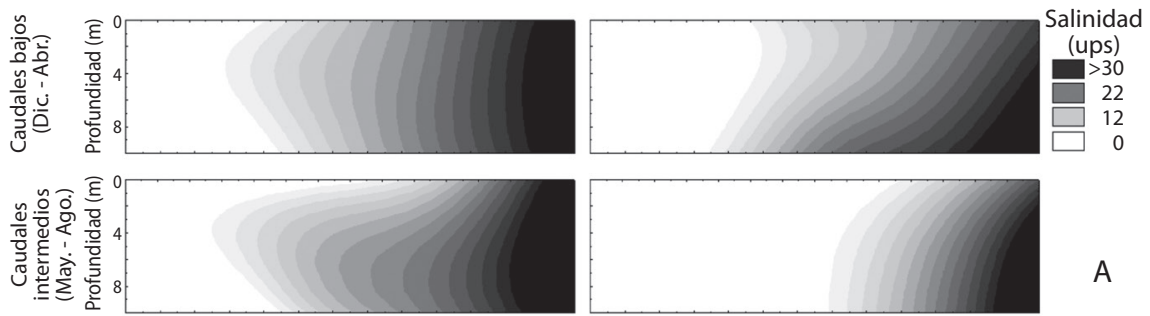

A
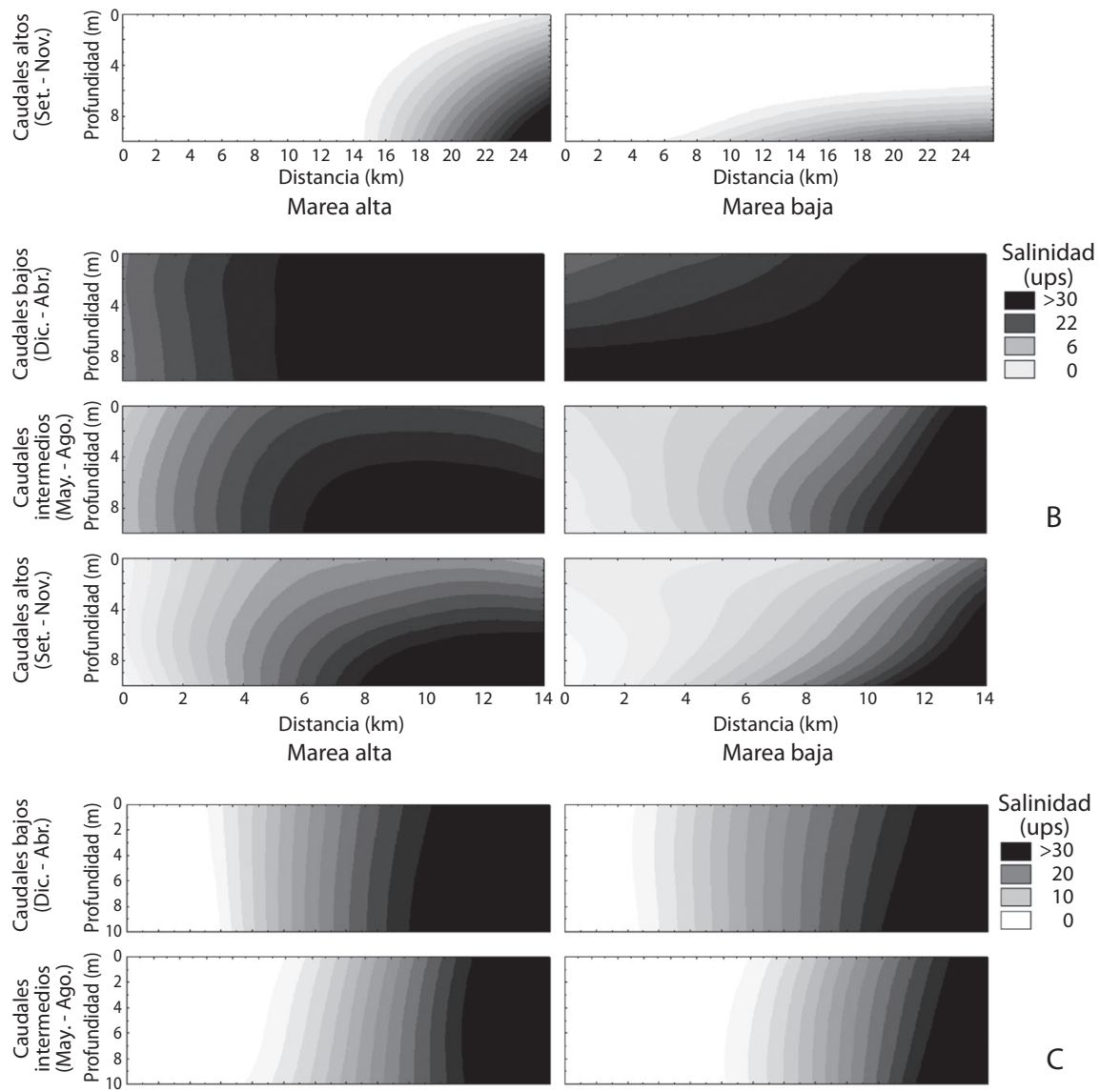

C
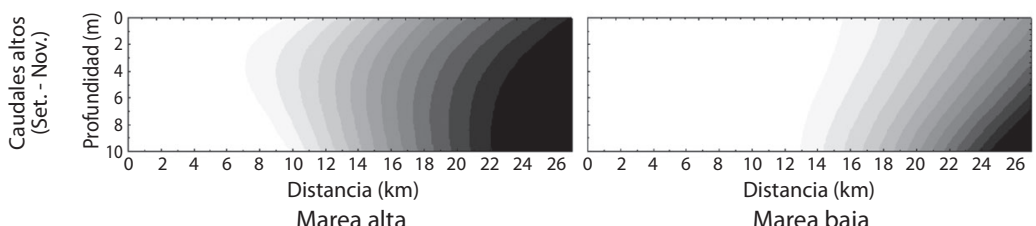

Fig. 10. Perfil de distribución de la cuña salinidad durante marea alta, marea baja y caudales bajos, intermedios y altos. A) sector norte desde Ciudad Cortés $(0 \mathrm{~km})$ hasta Boca Coronado $(24 \mathrm{~km})$, B) sector central desde el estero Caballo $(0 \mathrm{~km})$ hasta la boca Zacate $(14 \mathrm{~km})$, C) sector sur desde la comunidad de Sierpe $(0 \mathrm{~km})$ hasta la Boca Sierpe $(26 \mathrm{~km})$. ICE-PHED, periodo 2006-2012. El modelo fue ajustado a una profundidad promedio de $10 \mathrm{~m}$ a lo largo del perfil.

Fig. 10. Salt-wedge distribution during high and low tide, and during low, intermediate and high flows. A) northern sector profile from Ciudad Cortes $(0 \mathrm{~km})$ to Boca Coronado $(24 \mathrm{~km}), \mathrm{B})$ central sector profile from Caballo estuary $(0 \mathrm{~km})$ to Boca Zacate $(14 \mathrm{~km})$, C) southern sector profile from Sierpe town $(0 \mathrm{~km})$ to Boca Sierpe $(26 \mathrm{~km})$. ICE-PHED, period $2006-2012$. The model was adjusted to a $10 \mathrm{~m}$ deep water column throughout the profile. 
empezando a registrar valores bajos de salinidad (<5ups) a partir de los $14 \mathrm{~km}$ aguas abajo, mientras que los valores mayores a 15 ups se ubican muy cerca de la desembocadura (Fig. 10). La condición extrema de la influencia de los aportes de agua dulce durante los meses de setiembre-noviembre y la marea baja produce que prácticamente toda la columna de agua sea agua dulce ( $<1$ ups), y es hasta muy cerca de la desembocadura, $16 \mathrm{~km}$ aguas abajo, donde se inicia a registrar alguna influencia de agua marina pero con valores bajos de salinidad, que comprenden entre 2 ups y 8 ups en las capas del fondo. Por su parte durante la marea alta la influencia de la salinidad se observa a partir de los $16 \mathrm{~km}$ de distancia desde Ciudad Cortés con valores entre los 5ups y 15 ups.

En el sector central se confirma la fuerte influencia marina, los valores promedio se mantienen sobre los 5ups durante la mayor parte del año en todo el sector. Esta condición prevalece aún durante el periodo de mayor aporte de agua dulce en los meses de setiembre a noviembre. Como es de esperar, durante la marea alta la distribución de la salinidad en la columna de agua es bastante homogénea y durante la marea baja hay una tendencia a formar una cuña salina. Por su parte, el modelo generado para el sector sur confirma la presencia de un gradiente de incremento de la salinidad bien definido, y una distribución marcadamente homogénea de la salinidad en la columna de agua a partir del kilómetro seis hacia la desembocadura del Río Sierpe. También se observa una leve tendencia a generar una cuña salina durante el periodo de mayor aporte de agua dulce por parte del Río Sierpe (Fig. 10).

\section{DISCUSIÓN}

La evidencia recopilada sobre la distribución de la salinidad en el HNTS permite diferenciar tres zonas en el sector estuarino: la primera corresponde al extremo norte, dominado por el régimen fluvial impuesto por el Río Grande de Térraba, cuya influencia abarca los esteros y las bocas o desembocaduras de Coronado, Brava, Nueva y Chica y durante los caudales altos abarca al estero Estirahuevos. La segunda es una zona central que presenta una mayor influencia marina y caracterizada por una proyección del oleaje hacia el interior de los esteros Zacate, Caballo y Guarumal. La tercera zona corresponde al estero y desembocadura bajo la influencia del Río Sierpe, la cual mantiene una gradiente más estable de salinidad durante la mayor parte del año. Sin embargo dado el menor caudal del Río Sierpe, en comparación al Río Grande de Térraba y cuyas cuencas de drenaje poseen una relación de 6:1, la intrusión salina alcanza hasta la zona de los atracaderos en el poblado de Sierpe aproximadamente unos $30 \mathrm{~km}$ desde la desembocadura al mar.

De acuerdo al esquema de clasificación de los estuarios basado en la distribución vertical de la salinidad (Pritchard, 1955; Cameron \& Pritchard, 1963; Dyer, 1973), la zona norte del sector estuarino del HNTS presenta características que se asemejan a un estuario parcialmente mezclado durante los periodos de caudales bajos (diciembre-abril), el cual se caracteriza por la mezcla parcial entre el agua dulce y la salada producida probablemente por la turbulencia generada por la fricción entre las capas de agua con el fondo de los canales. Durante el periodo de caudales intermedios (mayo-agosto) el estuario tiende a ser estratificado y se asemeja a un estuario de cuña salina, en el cual típicamente las dos masas de agua se encuentran separadas, el agua dulce al ser menos densa que el agua salada fluye hacia el mar sobre la superficie de la capa de agua salada hasta las desembocaduras o bocas de Coronado, Nueva, Brava y Chica. Con la llegada de los caudales altos entre los meses de setiembre y noviembre se observa también un estuario tipo cuña salina pero con un alejamiento muy marcado hacia la desembocadura. Es probable que el efecto del agua dulce se manifieste en las capas superficiales fuera del sector estuarino del HNTS hasta el sector costero o Marino Deltaico en la bahía de Coronado. Sin embargo, aún en este periodo el estuario experimenta cambios en su volumen con las mareas, causando en muchas ocasiones desbordamientos en Ciudad 
Cortés. La zona central, al presentar un flujo de mareas más grande que el flujo de agua dulce posee características típicas de un estuario bien mezclado o estuario verticalmente homogéneo, en el cual la mezcla de la columna de agua se favorece por un intervalo de mareas mayor en relación con la profundidad del agua y la turbulencia producida por la tensión en el fondo (Dyer, 1973; Svendsen, 1997; Schoellhamer, 2001; Talley, 2002; Telesh \& Khlebovich, 2010). Esta condición se mantiene durante la mayor parte del año, si bien con el incremento en las precipitaciones hay un mayor aporte de agua dulce a esta zona no es suficiente para contrarrestar la acción de las mareas, además en esta zona es típico encontrar una fuerte influencia de vientos desde la costa hacia la parte interna de los esteros lo cual favorece la mezcla de la columna de agua. Por su parte el extremo sur del estuario se mantiene parcialmente mezclado durante la mayor parte del año, lo cual se asocia a un mayor equilibrio entre el caudal aportado por el Río Sierpe y la acción de las mareas.

Se debe tener presente que la salinidad en los estuarios es resultado del balance dinámico que varía en relación a procesos y fenómenos atmosféricos que controlan la circulación de las masas de agua (Telesh \& Khlebovich, 2010). A escalas anuales estacionales el agua dulce que llega por los ríos es el principal control de la distribución de la salinidad y la circulación en los estuarios (Sarma et al., 2009), mientras que variaciones en el tiempo atmosférico a escalas temporales mayores, como es el caso de los fenómenos de El Niño y La Niña, modifican el régimen habitual de precipitación y la descarga de agua dulce que entra a los estuarios, incidiendo directamente sobre la distribución estacional de la salinidad (Steinitz, Riedinger, Last, Brenner \& Miller, 1998; Schmidt \& Luther 2002; Vieira, Miranda \& Grimm, 2008).

De esta manera, el incremento en las precipitaciones asociado a la fase fría o La Niña en la costa Pacífica provoca descensos en los valores de salinidad en los estuarios, en contraparte durante la fase cálida o El Niño la reducción en las precipitaciones y eventualmente en los caudales tiende a favorecer el incremento en los valores de la salinidad estuarina (Drexler \& Ewel, 2001). En el área de estudio, utilizando datos de la estación hidrológica Palmar 31-01 del ICE durante el periodo 1963-2009, se observa una variación promedio del $10 \%$ con respecto al caudal promedio anual aportado por el Río Grande de Térraba al HNTS durante eventos de El Niño y La Niña, sin embargo pueden manifestarse condiciones extremas que producen diferencias de hasta un $40 \%$ del caudal promedio anual aportado al HNTS, como fue el caso de El Niño de 1992 y La Niña de 1996 (Zúñiga, 2005; Villalobos \& Retana, 2009, 2013; Solano \& Stolz, 2013).

Además, no se puede dejar de lado que existen procesos locales que causan variaciones a escalas temporales menores, como ocurrió entre los años 2005-2006 con la apertura de un nuevo canal en el sector de la isla Viuda durante una de las crecidas del Río Grande de Térraba durante la época de lluvias, lo cual provocó que el Río Grande de Térraba disminuyera su influencia horizontal en el sector estuarino y ahora siga una ruta más directa hacia el mar. Este proceso provocó cambios importantes en la distribución del agua dulce y, por ende, en la salinidad en varios esteros, por ejemplo los sitios boca Coronado, estero el Caite y boca Zacate durante el periodo 2000-2003 mantenían condiciones estuarinas similares donde predominaba la influencia marina (Picado et al., 2008), mientras que el canal Estirahuevos prevalecían características más fluviales evidenciando una fuerte influencia del Río Grande de Térraba. Posteriormente los datos recopilados por el laboratorio de química del ICE entre el 2007-2010 sugieren que el canal Estirahuevos ahora se comporta en una forma similar a la boca Zacate y la boca Coronado y que posee poca influencia del Río Grande de Térraba, mientras que el estero el Caite ahora presenta condiciones fluviales similares al sitio isla Viuda. Estos cambios físicos y químicos condujeron también a cambios en la composición y distribución de las especies del área, en particular la flora riparia la cual 
continúa experimentando procesos de colonización hacia el sector del estero el Caite (Picado et al., 2008).

Lo anterior es sólo un ejemplo de la marcada dinámica que existe en el sector estuarino del HNTS bajo la influencia directa del Río Grande de Térraba. Es de esperar que estos y futuros cambios provoquen ajustes en la abundancia y distribución en la flora y fauna, y la productividad biológica en este sector (Ingole \& Parulekar, 1998; Barletta, Barletta, Saint \& Hubold, 2005; Shan, RenChao, SuiSui \& SuHua, 2008; Sarma et al., 2009), y que este tipo de ajustes en realidad los ha venido experimentado el sistema en forma cíclica y a escalas temporales en el orden de décadas (Donguy \& Henin, 1976; Mann \& Lazier, 1996; Lalli \& Parsons, 2000; Talley, 2002; Stott et al., 2004; Robert, Schmitz, Kirauni \& Koedam, 2010).

La eventual construcción del Proyecto Hidroeléctrico El Diquís modificaría el régimen fluvial que entra al HNTS a través del Río Grande de Térraba. Es importante aclarar que el momento y cantidad de agua dulce aportada por parte de una central de generación es una función directa de disponibilidad del agua en el embalse y la demanda de energía en el país; tradicionalmente entre las $12 \mathrm{am}-5 \mathrm{am}$ se tiene la menor demanda y los máximos se concentran entre las $10 \mathrm{am}-1 \mathrm{pm}$ y entre $6 \mathrm{pm}-9 \mathrm{pm}$. Estos ajustes en la generación podrían implicar que en el caso del PHED se libere entre $62.5 \mathrm{~m}^{3} / \mathrm{s}$ y $250 \mathrm{~m}^{3} / \mathrm{s}$ en diferentes momentos, inclusive en un mismo día. Tomando en cuenta lo anterior, se prevé que el principal cambio en los aportes de agua dulce se presente durante los meses de caudales bajos (diciembre-abril), el cual podría oscilar entre los $122 \mathrm{~m}^{3} / \mathrm{s}$ y $396 \mathrm{~m}^{3} / \mathrm{s}$ dependiendo de la demanda y generación de electricidad. Bajo el escenario de generación mínima se reproduciría una condición similar al promedio actual de este periodo con un caudal de $115 \mathrm{~m}^{3} / \mathrm{s}$. Sin embargo, esto representaría un incremento del $82 \%$ en comparación con el aporte mínimo promedio de agua dulce, mientras que durante la generación máxima se aportaría un caudal similar al promedio del mes de junio que corresponde a $399 \mathrm{~m}^{3} / \mathrm{s}$, lo cual representaría un incremento del $66 \%$ en el aporte máximo promedio de agua dulce durante el periodo de caudales bajos. De este modo, se espera que los ciclos de marea alta que coincidan con los momentos de generación mínima mantengan una distribución de la salinidad similar al promedio actual en la época seca, mientras que durante la generación máxima de electricidad se observaría una disminución en la salinidad por procesos de dilución con el agua dulce aportada y que se asemeja a los meses de caudales intermedios.

Durante el periodo de caudales intermedios (mayo-agosto) se podrían presentar aportes mínimos de $216 \mathrm{~m}^{3} / \mathrm{s}$ y máximos de $462 \mathrm{~m}^{3} / \mathrm{s}$ por la operación del proyecto. Esto causaría una ampliación en el ámbito promedio de caudales registrados actualmente, cuyo mínimo es de $281 \mathrm{~m}^{3} / \mathrm{s}$ y máximo de $399 \mathrm{~m}^{3} / \mathrm{s}$, lo cual representa una disminución en un $23 \%$ del aporte mínimo promedio de agua dulce y un incremento del $16 \%$ en el aporte máximo promedio. En el caso del periodo de caudales altos (setiembre-noviembre) se podrían presentar aportes mínimos de $345 \mathrm{~m}^{3} / \mathrm{s}$ y máximos de $632 \mathrm{~m}^{3} / \mathrm{s}$, en este caso se reducirían tanto el límite inferior y el límite superior del ámbito de caudales promedio registrados durante este periodo, esto representa una disminución en los aportes de agua dulce de un 37\% del aporte mínimo promedio y de un $18 \%$ del aporte máximo promedio. Estos posibles escenarios de aportes de agua dulce durante estos dos últimos periodos están contenidos dentro de la variabilidad natural del sistema, por esta razón no se prevé un cambio significativo, en este momento, en la distribución de la salinidad con la posible puesta en operación del PHED.

Dado lo anterior, se podría esperar que en la zona norte del sector estuarino del HNTS prevalezcan características de un estuario de cuña salina durante la mayor parte del año, esto como respuesta a un incremento en los aportes de agua dulce durante los meses de diciembremayo y una disminución en los aportes de agua dulce durante los meses de setiembrenoviembre. Aún bajo este nuevo régimen, que es más equilibrado o estable a lo largo del año 
que el actual, no se prevé un cambio en la distribución de la salinidad en los primeros siete kilómetros aguas abajo de Ciudad Cortés, ya que los caudales mínimos que se podrían generar con la operación del PHED son superiores a los mínimos de este periodo, lo cual garantiza que continúe manteniendo sus características fluviales, al menos en términos de los valores de salinidad.

Cabe mencionar que los escenarios descritos corresponden a los límites mínimos y máximos promedio que se podrían presentar en relación con una generación de energía eléctrica a la mínima o máxima capacidad. Entre estos valores existen múltiples puntos intermedios, así como escenarios extremos o críticos. Sin embargo, tampoco se debe dejar de lado el hecho de que el sector estuarino del HNTS es dinámico y en forma natural está sujeto a cambios inclusive diarios en la salinidad a partir de los aportes de los ríos y el ciclo de las mareas. El presente trabajo representa uno de los primeros pasos hacia el entendimiento de la dinámica estuarina del HNTS. Es necesario complementar los análisis con modelos numéricos predictivos así como como vincular los resultados con el componente biológico, información que será presentada en futuras entregas. El monitoreo de la salinidad y otros parámetros biofísicos, es esencial para poder discernir en el futuro entre las posibles fuentes de cambios en el sector estuarino del HNTS y deberá ser considerado como un compromiso firme por parte de las instituciones involucradas en el manejo y conservación de este ecosistema.

\section{AGRADECIMIENTOS}

Se agradece el apoyo y dedicación del personal de campo de las áreas de Hidrología y Biología del proyecto Hidroeléctrico El Diquís, en especial a Leonel Barquero y Carlos Canales, así como al personal del laboratorio de Química de Hidrología del ICE por su contribución con la colecta y análisis físicos y químicos de las muestras de agua del HNTS. A Freddy Centeno por toda su colaboración en el manejo de las bases de datos y elaboración de los mapas.

\section{RESUMEN}

El análisis de la salinidad durante tres campañas de monitoreo (2002-2004, 2005-2008 y 2011-2012), permite establecer que el sector estuarino del HNTS se divide en una zona norte con una fuerte influencia del Río Grande de Térraba y características de un estuario parcialmente mezclado durante el periodo de caudales bajos (diciembreabril), mientras que se asemeja a un estuario estratificado de cuña salina durante los caudales intermedios (mayoagosto) y caudales altos (setiembre-noviembre). La zona central, con una mayor influencia marina, presenta características de un estuario bien mezclado o verticalmente homogéneo, mientras que la zona sur del estuario, bajo la influencia del Río Sierpe, se mantiene parcialmente mezclado a lo largo del año. Los primeros siete kilómetros aguas abajo de Ciudad Cortés presentaron una condición fluvial muy bien definida con salinidades inferiores a 1ups, a partir del kilómetro 14 se observa un incremento gradual de los valores de salinidad hasta 30ups hacia la desembocadura, reflejando una mayor influencia de las mareas en este último tramo $\left(\mathrm{F}_{1,47}=2.5, \mathrm{p}<0.001\right)$. El eventual desarrollo del Proyecto Hidroeléctrico El Diquís podría ocasionar un incremento en el aporte de agua dulce entre un 82 y $66 \%$ de los valores mínimos promedio y máximos promedio sobre la zona norte del sector estuarino del HNTS durante el periodo de caudales bajos, lo cual generaría condiciones semejantes a las registradas durante el periodo de caudales intermedios. El monitoreo de las relaciones entre los aspectos físicos-químicos y biológicos es esencial para entendimiento de los posibles cambios en el sector estuarino del HNTS.

Palabras clave: Salinidad, estuario, humedal, Térraba, Sierpe, Diquís.

\section{REFERENCIAS}

Acuña, G. J., Vargas, J. A., \& Córdoba, R. (2006). A snapshot view of some vertical distributions of water parameters at a deep $(200 \mathrm{~m})$ station in the fjord-like Golfo Dulce, embayment, Costa Rica. Revista de Biología Tropical, 54(Suppl. 1), 193-200.

Barletta, M., Barletta, A., Saint, U., \& Hubold, G. (2005). The role of salinity in structuring the fish assemblages in a tropical estuary. Journal of Fish Biology, $66,45-72$.

Bravo, J., \& Ocampo, L. (1993). Mapa de humedales de Costa Rica. Escala 1:750.000. Costa Rica: Universidad Nacional, Unión Internacional para la Conservación de la Naturaleza (UICN).

Brenes, C. L., León, S., \& Chaves. J. (2001). Variación de las propiedades termohalinas en el Golfo de Nicoya, Costa Rica. Revista de Biología Tropical, 49(2 Suppl.), 145-152. 
Cameron, W. M., \& Pritchard, D. W. (1963). Estuaries. In M. N. Hill (Ed.), The Sea (Vol. 2, pp. 306-324). NewYork: Wiley.

Clarke, K. R., \& Warwick, R. M. (1994). Change in marine communities: An approach to statistical analysis and interpretation. United Kingdom: Natural Environment Research Council.

Cordero, P. P. (2000). El manglar más grande de Costa Rica: experiencias de la UICN en el proyecto Danida-Manglares de Térraba-Sierpe. San José, Costa Rica: UICN.

Donguy, J. R., \& Henin, C. (1976). Anomalous navifacial salinities in the tropical Pacific Ocean. Journal of Marine Research, 34, 355-364.

Drexler, Z. J., \& Ewel, K. C. (2001). Effect of the 19971998 ENSO-Related Drought on Hydrology and Salinity in a Micronesian Wetland Complex. Estuaries, 24(3), 347-356.

Dyer, K. R. (1973). Estuaries: a physical introduction. London: Wiley.

Fonseca, A. C., Alvarado J. J., Cortés, J., \& Herrera, B. (2009). Planificación marino-costera del Área de Conservación Osa (ACOSA), Costa Rica. San José, Costa Rica: Serie Técnica Apoyando los esfuerzos en el manejo y protección de la biodiversidad tropical. No. 12. TNC.

Gómez, R. E. (2006). Ambientes Marino Costeros de Costa Rica. Informe Técnico. In V. Nielsen \& M. A. Quesada (Eds.), Estuarios (pp. 11-16). San José, Costa Rica: Comisión Interdisciplinaria Marino Costera de la Zona Económica Exclusiva de Costa Rica.

Hammer, Ø., Harper, D. A. T., \& Ryan, P. D. (2001). PAST. Paleontological statistics software package for education and data analysis. Palaeontologia Electronica, 4(1), 9. Recuperado de http://palaeo-electronica. org/2001 1/past/issue1 01.htm

Ingole, B. S., \& Parulekar, A. H. (1998). Role of salinity in structuting the intertidal meiofauna of a tropical estuarine beach: field evidence. Indian Journal of Marine Sciences, 27, 356-361.

Jiménez, J. A. (1994). Los manglares del Pacífico de Centroamérica. Heredia, Costa Rica: Editorial Fundación UNA.

Kapelle, M., Acevedo, M., González, L., \& Monge, H. (2002). Ecosistemas del Área de Conservación Osa ( ACOSA). Heredia, Costa Rica: Instituto Nacional de Biodiversidad (INBio).

Krebs, C. J. (1994). Ecological methodology. USA: Benjamin Cummings, Addison Wesley Longman.

Lalli, C. M., \& Parsons, T. R. (2000). Biological oceanography: an introduction. 2nd ed. Great Britain: Butterworth-Heinemann.
Lizano, O. G. (1997). Las mareas extraordinarias de 1997 en la costa del Pacífico de Costa Rica. Tópicos Meteorológicos y Oceanográficos, 4(2), 169-179.

Lizano, O. G., \& Vargas, J. A. (1993). Distribución espaciotemporal de la salinidad y la temperatura en la parte interna del Golfo de Nicoya, Costa Rica. Tecnología en Marcha, 12, 3-16.

Lizano, O. G. (2006). Algunas características de las mareas en la costa Pacífica y Caribe de Centroamérica. Ciencia y tecnología, 24(1), 51-64.

Mann, K. H., \& Lazier, J. R. N. (1996). Dynamics of marine ecosystems. 2nd ed. Oxford: Blackwell Science.

Picado, B. J., Lara H., Castro, D. V., \& Barrantes, L. (2008). Caracterización Biofísica del Humedal Nacional Térraba Sierpe. (Informe Técnico-PHED). Costa Rica: Instituto Costarricense de Electricidad.

Pla, L., \& Matteucci, S. D. (2001). Intervalos de confianza "bootstrap" del índice de biodiversidad de Shannon. Revista de la Facultad de Agronomía, 18, 222-234.

Pritchard, D. W. (1955). Estuarine circulation patterns. Proceedings of the American Society of Civil Engineers, 81(717), 1-11.

Robert, E., Schmitz, N., Kirauni, H. A., \& Koedam, N. (2010). Salinity fluctuations in mangrove forest of Gazi bay, Kenya: lessons for future research. Nature and Faune, 24(1), 89-95.

Robertson, A. I., \& Alongi, D. M. (1992). Tropical Mangrove Ecosystems. Washington, USA: American Geophysical Union.

Rojas, N. (2011). Estudio de Cuencas Hidrográficas de Costa Rica: Cuenca del Río Grande de Térraba. San José, Costa Rica: Instituto Meteorológico Nacional.

Sarma, V. V. S. S., Gupta, S. N. M., Babu, P. V. R., Acharya, T., Harikrishnachari, N., Vishnuvardhan, ... Kumar, M. D. (2009). Influence of river discharge on plankton metabolic rates in the tropical monsoon driven Godavari estuary, India. Estuarine, Coastal and Shelf Science, 85, 515-524.

Shan, L., RenChao, Z., SuiSui, D., \& SuHua, S. (2008). Adaptation to salinity in Mangroves: Implication on the evolution of salt-tolerance. China Science Bulletin, 53(11), 1708-1715.

Sierra, C., Vartanián, D., \& Polimeni, J. (2006). Caracterización Social, Económica y Ambiental del Área de Conservación de Osa. (2da ed). San José, Costa Rica: Dirección de Sociedad Civil, Ministerio del Ambiente y Energía.

Silva, A. M., \& Acuña, J. (2006). Caracterización físicoquímica de dos estuarios en la bahía de Golfito, Golfo Dulce, Pacífico de Costa Rica. Revista de Biología Tropical, 54(1 Suppl.), 241-256. 
Schmidt, N., \& Luther, M. E. (2002). ENSO impacts on salinity in Tampa Bay, Florida. Estuaries, 25(5), 976-984.

Schoellhamer, D. H. (2001). Influence of salinity, bottom topography, and tides on locations of estuarine turbidity maxima in northern San Francisco Bay. In W. H. McAnally \& A. J. Mehta (Eds.), Coastal and Estuarine Fine Sediment Transport Processes: Elsevier Science B.V (pp. 343-357). Retrieved from http:// ca.water.usgs.gov/abstract/sfbay/elsevier0102.pdf.

Solano, E., \& Stolz, W. (2013). El Fenómeno ENOS (El NIÑO/Oscilación del Sur). Recuperado de http:// www.imn.ac.cr/educacion/enos.html

Steinitz, K. M., Riedinger, M. A., Last, W., Brenner, M., \& Miller, M. C. (1998). Un registro de 6000 años de manifestaciones intensas del Fenómeno de El Niño en sedimentos de lagunas de las islas Galápagos. Bulletin de l'Institut Français d'Etudes Andines, 27(3), 581-592.

Stott, L., Cannariato, K., Thunell, R., Haug, G. H., Koutavas, A., \& Lund, S. (2004). Decline of surface temperature and salinity in the western tropical Pacific Ocean in the Holocene epoch. Nature, 431, 56-59.

Svendsen, H. (1997). Physical oceanography and marine ecosystems: some illustrative examples. Scientia Marina, 61(1), 93-108.

Svendsen, H., Rosland, R., Myking, S., Vargas, J. A., Lizano, O. G., \& Alfaro, E. J. (2006). A physicaloceanographic study of Golfo Dulce, Costa Rica. Revista de Biología Tropical, 54(1 Suppl.), 147-170.

Talley, L. D. (2002). Salinity Patters in the ocean. In M. C. MacCracken \& J. S. Perry (Eds.), The Earth system: physical and chemical dimensions of global environmental change (pp. 629-640). Chichester, Ecngland: John Wiley \& Sons, Ltd.

Telesh, I. V., \& Khlebovich, V. V. (2010). Principal processes within the estuarine salinity gradient: A review. Marine Pollution Bulletin, 61, 149-155.

Umaña, G., Bolaños, F., Mora, J., Pereira, A., \& Gómez, J. (2000). Inventario de las especies que habitan en el manglar de Térraba-Sierpe y estado actual del ecosistema. Costa Rica: Escuela de Biología, Universidad de Costa Rica.

Vieira, P. J., Miranda, A. G., \& Grimm, A. M. (2008). Evidences of El Niño Effects on the Mullet Fishery of the Patos Lagoon Estuary. Brazilian Archives of Biology and Technology, 51(2), 433-440.

Villalobos, R., \& Retana, J. A. (2009). El Niño: una revisión bibliográfica. Recuperado de http://www.imn. ac.cr/publicaciones/estudios/Nino_rev_bibliog.pdf.

Villalobos, R., \& Retana, J. A. (2013). Caracterización pluviométrica de la fase cálida de ENOS en Costa Rica con base en probabilidades de ocurrencia de eventos en tres escenarios: seco, normal y lluvioso. Recuperado de http:/www.imn.ac.cr/publicaciones/ estudios/Nino_prob_lluvia_escenarios.pdf

Voorhis, A., Epifanio, C., Maurer, D., Dittel, A., \& Vargas, J. (1983). The estuarine character of the Gulf of Nicoya, an embayment on the Pacific coast of Central América. Hydrobiologia, 99, 225-237.

Zar, J. H. (1999). Biostatistical analysis. (4th ed.). USA: Prentice Hall.

Zúñiga, J. A. (2005). Informe hidrológico del Proyecto Veraguas (Informe Técnico-Estudios Básicos). San José, Costa Rica: Instituto Costarricense de Electricidad. 